\title{
MELT \\ a Translated Domain Specific Language Embedded in the GCC Compiler
}

\author{
Basile STARYNKEVITCH \\ CEA, LIST \\ Software Safety Laboratory, boîte courrier 94, 91191 GIF/YvETTE CEDEX, France \\ basile@starynkevitch.net basile.starynkevitch@cea.fr
}

\begin{abstract}
The GCC free compiler is a very large software, compiling source in several languages for many targets on various systems. It can be extended by plugins, which may take advantage of its power to provide extra specific functionality (warnings, optimizations, source refactoring or navigation) by processing various GCC internal representations (Gimple, Tree, ...). Writing plugins in C is a complex and time-consuming task, but customizing GCC by using an existing scripting language inside is impractical. We describe $M E L T$, a specific Lisp-like DSL which fits well into existing GCC technology and offers high-level features (functional, object or reflexive programming, pattern matching). MELT is translated to $\mathrm{C}$ fitted for GCC internals and provides various features to facilitate this. This work shows that even huge, legacy, software can be a posteriori extended by specifically tailored and translated high-level DSLs.
\end{abstract}

\section{Introduction}

$\mathrm{GCC}^{1}$ is an industrial-strength free compiler for many source languages $(\mathrm{C}, \mathrm{C}++$, Ada, Objective $\mathrm{C}$, Fortran, Go, ...), targetting about 30 different machine architectures, and supported on many operating systems. Its source code size is huge $\left(4.296 \mathrm{MLOC}^{2}\right.$ for GCC 4.6.0), heterogenous, and still increasing by $6 \%$ annually 3 . It has no single main architect and hundreds of (mostly full-time) contributors, who follow strict social rules 4

\subsection{The powerful GCC legacy}

The several GCC [8] front-ends (parsing C, C++, Go ... source) produce common internal AST (abstract syntax tree) representations called Tree and Generic. These are later transformed into middle-end internal representations, the Gimple statements - through a transformation called gimplification. The bulk of the compiler is its middle-end which operates repeatedly on these Gimple representations 5 . It contains nearly 200 passes moulding these (in different forms). Finally, back-ends (specific to the target) work on Register Transfer Language (RTL) representations and emit assembly code. Besides that, many other

\footnotetext{
${ }^{1}$ Gnu Compiler Collection (gcc 4.6.0 released on march $25^{\text {th }} 2011$ ) on gcc.gnu.org

24.296 Millions Lines Of source Code, measured with David Wheeler's SLOCCount. Most other tools give bigger code measures, e.g., ohcount gives 8.370MLOC of source, with 5.477MLOC of code and 1.689MLOC of comments.

${ }^{3}$ GCC 4.4 .1 , released July $22^{\text {th }}, 2009$, was 3.884 MLOC, so a 0.412 MLOC $=10.6 \%$ increase in 1.67 years

${ }^{4}$ Every submitted code patch should be accepted by a code reviewer who cannot be the author of the patch, but there is no project leader or head architect, like Linus Torvalds is for the Linux kernel. So GCC has not a clean, well-designed, architecture.

${ }^{5}$ The GCC middle-end does not depend upon the source language or the target processor (except with parameters giving sizeof (int) etc.).
}

Olivier Danvy, Chung-chieh Shan (Eds.): IFIP Working Conference on Domain-Specific Languages 2011 (DSL 2011).

EPTCS 66, 2011, pp. 118-142 doi 10.4204/EPTCS.66.6 (c) B.Starynkevitch

This work is licensed under the Creative Commons Attribution-Share Alike License. 
data structures exist within GCC (and a lot of global variables). Most of the compiler code and optimizations work by various transformations on middle-end internal representations. GCC source code is mostly written in $C$ (with a few parts in $\mathrm{C}++$, or Ada), but it also has several internal $C$ code generators. GCC does not use parser generators (like flex, bison, etc).

It should be stressed that most internal GCC representations are constantly evolving, and there is no stability ${ }^{6}$ of the internal GCC API 7 . This makes the embedding of existing scripting languages (like Python, Ocaml, ...) impractical $(\$ 1.2)$. Since gcc 4.5 it is possible to enhance GCC through external plugins.

External plugins can enhance or modify the behavior of the GCC compiler through a defined interface, practically provided by a set of $C$ file headers, and made of functions, many $C$ macros, and coding conventions. Plugins are loaded as dlopen-ed dynamic shared objects at gcc run time. They can profit from all the variety and power of the many internal representations and processing of GCC. Plugins enhance GCC by inserting new passes and/or by responding to a set of plugin events (like PLUGIN_FINISH_TYPE when a type has been parsed, PLUGIN_PRAGMAS to register new pragmas, ...).

GCC plugins can add specific warnings (e.g., to a library), specific optimizations (e.g., transform fprintf $($ stdout,...$) \rightarrow \operatorname{printf}(. .$.$) in user code with \#include <stdio.h>), compute software metrics,$ help on source code navigation or code refactoring, etc. GCC extensions or plugins enable using and extending GCC for non code-generation activities like static analysis [9, 2, 17, 28], threats detection (like in Two [10], Coverity ${ }^{\mathrm{TM}} 8$, or Astrée[4, 5]), code refactoring, coding rules validation[16], etc. They could provide any processing taking advantage of the many facilities already existing inside GCC. However, since coding GCC plugins in $C$ is not easy, a higher-level DSL could help. Because GCC plugins are usually specific to a narrow user community, shortening their development time (through a higher-level language) makes sense.

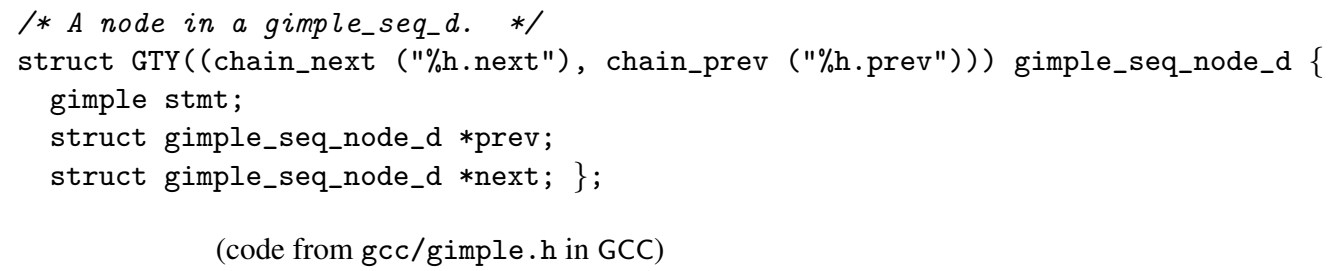

Figure 1: example of GTY annotation for $\mathrm{Gg}-\mathrm{c}$

Since compilers handle many complex (perhaps circular) data structures for their internal representations, explicitly managing memory is cumbersome during compilation. So the GCC community has added a crude garbage collector [11] Gg-c (GCC Garbage Collector): many $C$ struct-ures in GCC code are annotated with GTY (figure 1 ) to be handled by Gg-c; passes can allocate them, and a precise 9 mark and sweep garbage collection may be triggered by the pass manager only between passes. Gg-c does not know about local pointers, so garbage collected data is live and kept only if it is (indirectly) reachable from known global or static GTY-annotated variables (data reachable only from local variables would be lost). Data internal to a GCC pass is usually manually allocated and freed. GTY annotations on types and

\footnotetext{
${ }^{6}$ This is nearly a dogma of its community, to discourage proprietary software abuse of GCC.

${ }^{7}$ GCC has no well defined and documented Application Programming Interface for compiler extensions; its API is just a big set of header files, so is a bit messy for outsiders.

${ }^{8}$ See www . coverity. com

${ }^{9} \mathrm{Gg}$-c is a precise G-C knowing each pointer to handle; using Boehm's conservative garbage collector with ambigous roots inside GCC has been considered and rejected on performance grounds.
} 
variables inside GCC source are processed by gengtype, a specialized generator (producing $C$ code for $\mathrm{Gg}-\mathrm{c}$ allocation and marking routines and roots registration). There are more than 1800 GTY-ed types known by Gg-c, such as: gimple (pointing to the representation of a Gimple statement), tree (pointer to a structure representing a Tree), basic_block (pointing the representation of a basic block of Gimple-s), edge (pointing to the data representing an edge between basic blocks in the control flow graph), etc. Sadly, not all GCC data is handled by Gg-c; a lot of data is still manually micro-managed. We call stuff all the GCC internal data, either garbage-collected and GTY-annotated like gimple, tree, .., or outside the heap like raw long numbers, or even manually allocated like struct opt_pass (data describing GCC optimization passes).

GCC is a big legacy system, so its API is large and quite heterogenous in style. It is not only made of data declarations and functions operating on them, but also contains various $C$ macros. In particular, iterations inside internal representations may be provided by various styles of constructs:

1. Iterator abstract types like (to iterate on every stmt, a gimple inside a given basic block bb)

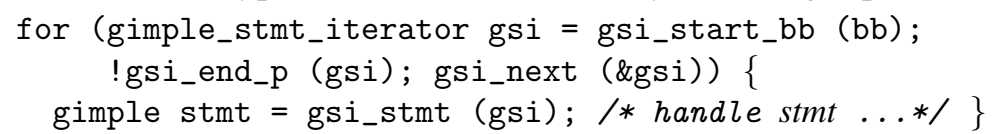

2. Iterative for-like macros, e.g., (to iterate for each basic block bb inside the current function cfun) basic_block bb; FOR_EACH_BB (bb) $\{/ *$ process $b b * /\}$

3. More rarely, passing a callback to an iterating "higher-order" $C$ function, e.g., (to iterate inside every index tree from ref and call idx_infer_loop_bounds on that index tree)

for_each_index (\&ref, idx_infer_loop_bounds, \&data);

with a static function bool idx_infer_loop_bounds (tree base, tree *idx, void *dta) called on every index tree base.

\subsection{Embedding an existing scripting language is impractical}

Interfacing GCC to an existing language implementation like Ocaml, Python, Guile, Lua, Ruby or some other scripting language is not realistic ${ }^{10}$, because of an impedance mismatch:

1. Most scripting languages are garbage collected, and mixing several garbage collectors is difficult and error-prone, in particular when both Gg-c and scripting language heaps are intermixed.

2. The GCC API is very big, ill-defined, heterogenous, and evolving significantly. So manually coding the glue code between GCC and a general-purpose scripting language is a big burden, and would be obsoleted by a new GCC version when achieved.

3. The GCC API is not only made of $C$ functions, but also of macros which are not easy to call from a scripting language.

4. Part of the GCC API is very low-level (e.g., field accessors), and would be invoked very often, so may become a performance bottleneck if used through a glue routine.

5. GCC handles various internal data (notably using hundreds of global variables), some through GTY-ed Gg-c collected pointers (like gimple_seq, edge, ...), others with manually allocated data (e.g., omp_region for OpenMP parallel region information) or with numbers mapping some opaque information (e.g., location_t are integers encoding source file locations). GCC data has widely different types, usage conventions, or liveness.

6. There is no single root type (e.g., a root class like GObject 11 in Gtk) which would facilitate gluing

\footnotetext{
${ }^{10}$ The author spent more than a month of work trying in vain to plug Ocaml into GCC!

${ }^{11}$ See http://developer.gnome.org/gobject/
} 
GCC into a dynamically typed language interpreter (à la Python, Guile, or Ruby).

7. Statically typing GCC data into a strongly typed language with type inference like Ocaml or Haskell is impractical, since it would require the formalization of a type theory compatible with all the actual GCC code.

8. Easily filtering complex nested data structures is very useful inside compilers, so most GCC extensions need to pattern-match on existing GCC stuff (notably on Gimple or Tree-s).

The MELT (originally meaning "Middle End Lisp Translator”) Domain Specific Language has been developped to increase, as any high-level DSL does, the programmer's productivity. MELT has its specific generational copying garbage collector above $\mathrm{Gg}$-c to address point 1 . Oddity of the GCC API (points 2, 3, 4) is handled by generating well fit $C$ code, and by providing mechanisms to ease that $C$ source code generation. Items 4, 5, 6, 7 are tackled by mixing MELT dynamically typed values with raw GCC stuff. MELT has a powerful pattern matching ability to handle last point 8 , because scripting languages don't offer extensible or embeddable pattern matching (on data structures internal to the embedding application).

MELT is being used for various GCC extensions (work in progress):

- simple warning and optimization like fprintf (stdout ,...) detection and transformation (handling it on Gimple representation is preferable to simple textual replacement, because it cooperates with the compiler inlining transformation);

- Jérémie Salvucci has coded a Gimple $\rightarrow C$ transformer (to feed some other tool);

- Pierre Vittet is coding various domain-specific warnings (e.g., detection of untested calls to fopen);

- the author is developing an extension to generate OpenCL code from some Gimple, to transport some highly parallel regular (e.g., matrix) code to GPUs;

\subsection{MELT = a DSL translated to code friendly with GCC internals}

The legacy constraints given by GCC on additional (e.g., plugins') code suggest that a DSL for extending it could be implemented by generating $C$ code suitable for GCC internals, and by providing language constructs translatable into $C$ code conforming to GCC coding style and conventions. Other attempts to embed a scripting language into GCC (Javascript [9] for coding rules in Firefox, Haskell for enhancing $\mathrm{C}++$ template meta-programming [1], or Python ${ }^{12}$ ) have restricted themselves to a tiny part of the GCC API; Volanschi [29] describes a modified GCC compiler with specialized matching rules.

Therefore, the reasonable way to provide a higher-level domain specific language for GCC extensions is to dynamically generate suitable $\boldsymbol{C}$ code adapted to GCC's style and legacy and similar in form to existing hand-coded $C$ routines inside GCC. This is the driving idea of our MELT domain specific language and plugin implementation [24, 25, 26]. By generating suitable $C$ code for GCC internals, MELT fits well into existing GCC technology. This is in sharp contrast with the Emacs editor or the C-- compiler [23] whose architecture was designed and centered on an embedded interpreter (E-Lisp for Emacs, Lua $^{\text {ocaml }}$ for C--).

MELT is a Lisp-looking DSL designed to work on GCC internals. It handles both dynamically typed MELT values and raw GCC stuff (like gimple, tree, edge and many others). It supports applicative, object and reflective programming styles. It offers powerful pattern matching facilities to work on GCC internal representations, essential inside a compiler. It is translated into $C$ code and offer linguistic devices to deal nicely with GCC legacy code.

\footnotetext{
${ }^{12}$ See David Malcom's GCC Python plugin announced in http://gcc.gnu.org/ml/gcc/2011-06/msg00293.html
} 


\section{Using MELT and its runtime.}

\subsection{MELT usage and organization overview}

From the user's perspective, the GCC compiler enabled with MELT (GCC ${ }^{\text {melt }}$ ) can be run with a command as: gcc -fplugin=melt -fplugin-arg-melt-mode=opengpu $-0-c$ foo.c. This instructs gcc (the gcc-4.6 packaged in Debian) to run the compiler proper cc1, asks it to load the melt.so plugin which provides the MELT specific runtime infrastructure, and passes to that plugin the argument mode=opengpu while cc1 compiles the user's foo.c. The melt.so plugin initializes the MELT runtime, hence itself dlopen-s MELT modules like warmelt*. so \& xtramelt*. so. These modules initialize MELT data, e.g., classes, instances, closures, and handlers. The MELT handler associated to the opengpu mode registers a new GCC pass (available in xtramelt-opengpu.melt) which is executed by the GCC pass manager when compiling the file foo.c. This opengpu pass uses Graphite [27] to find optimization opportunities in loops and should ${ }^{13}$ generate OpenCL code to run these on GPUs, transforming the Gimple to call that generated OpenCL code. The melt.so plugin is mostly hand-coded in $\mathrm{C}$ (in our melt-runtime. [hc] files - 15KLOC, which \#include generated files). The MELT modules warmelt*. so \& xtramelt*.so 14 are coded in MELT (as source files warmelt*.melt, .., xtramelt*. melt which have been translated by MELT into generated C files warmelt*.c \& xtramelt-*.c, themselves compiled into modules warmelt*.so ...).

The MELT translator (able to generate *.c from *.melt) is bootstrapped so that it exercises most of its features and its runtime : the translator's source code is coded in MELT, precisely the melt/warmelt*.melt files (39KLOC), and the MELT source repository also contains the generated files melt/generated/warmelt*.c (769KLOC). Other MELT files, like melt/xtramelt*.melt (6KLOC) don't need to have their generated translation kept. The MELT translator ${ }^{15}$ is not a GCC front-end (since it produces C code for the host system, not Generic or Gimple internal representations suited for the target machine); and it is even able to dynamically generate, during an $\mathrm{GCC}^{\text {melt }}$ compiler invocation, some temporary *.c code, run make to compile that into a temporary *. so, and load (i.e. dlopen) and execute that - all this in a single gcc user invocation; this can be useful for sophisticated static analysis [25] specialized using partial evaluation techniques within the analyzer, or just to "run" a MELT file.

The MELT translator works in several steps: the reader builds s-expressions in MELT heap. Macroexpansion translates them into a MELT AST. Normalization introduces necessary temporaries and builds a normal form. Generation makes a representation very close to $C$ code. At last that representation is emitted to output generated $C$ code. There is no optimization done by the MELT translator (except for compilation of pattern matching, see $\$ 4.4$ ).

Translation from MELT code to C code is fast: on a $x 86-64 \mathrm{GNU} /$ Linux desktop system ${ }^{16}$, the $6.5 \mathrm{KLOC}$ warmelt-normal.melt file is translated into five warmelt-normal*.c files with a total of 239KLOC in just one second (wall time). But 32 seconds are needed to build the warmelt-normal.so module (with make ${ }^{17}$ running gcc $-01-f P I C$ ) from these generated $C$ files. So most of the time is spent in compiling the generated $\mathrm{C}$ code, not in generating it. In contrast to several DSLs persisting their

\footnotetext{
${ }^{13}$ In April 2011, the opengpu pass, coded in MELT, is still incomplete in MELT 0.7 svn rev.173182.

${ }^{14}$ The module names warmelt*.so \& xtramelt*. so are somehow indirectly hard-coded in melt-runtime.c but could be overloaded by many explicit -fplugin-arg-melt-* options.

${ }^{15}$ The translation from file ana-simple.melt to ana-simple.c is done by invoking gcc $-\mathrm{fplugin=melt}$ -fplugin-arg-melt-mode=translatefile -fplugin-arg-melt-arg=ana-simple.melt ...on an empty C file empty.c, only to have cc1 launched by gcc!

${ }^{16}$ An Intel Q9550 @ 2.83GHz, 8Gb RAM, fast 10KRPM Sata 150Gb disk, Debian/Sid/AMD64.

${ }^{17}$ So it helps to run that in parallel using make $-j$; the 32 seconds timing is a sequential single-job make.
} 


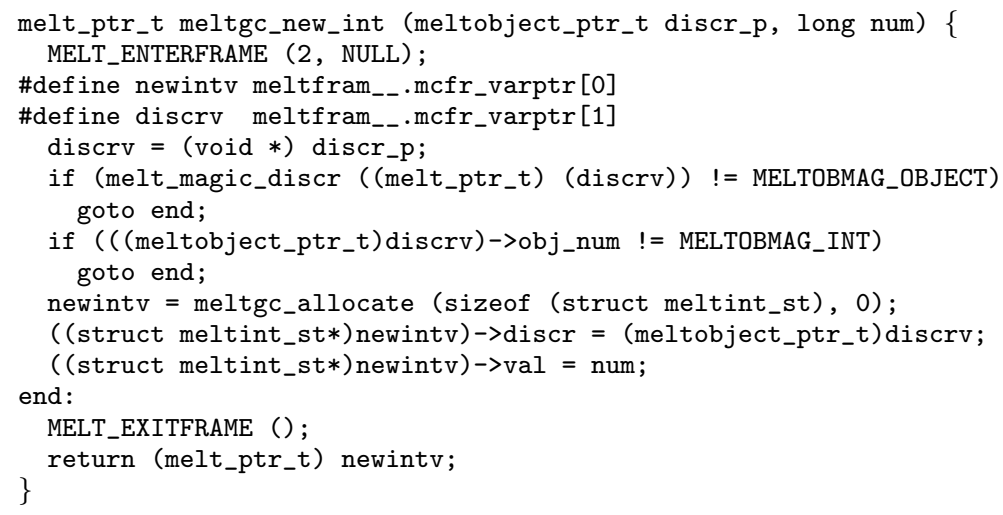

Figure 2: MELT runtime function boxing an integer

closures ${ }^{18}$ by serializing a mixture of data and code, MELT starts with an empty heap, so MELT modules' initialization routines are mostly long and sequential $\mathrm{C}$ code initializing the MELT heap.

\subsection{MELT runtime infrastructure}

The MELT runtime melt-runtime.c is built above the GCC infrastructure, notably Gg-c. However, Ggc is not a sufficient garbage collector for MELT values, like closures, lists, tuples, objects, ... As in most applicative or functional languages, MELT code tends to allocate a lot of temporary values (which often die quickly). So garbage collection (G-C) of MELT values may happen often, and does need to happen even inside GCC passes written in MELT, not only between passes. These values are handled by our generational copying MELT G-C, triggered by the MELT allocator when its birth region is full, and backed up by the existing Gg-c (so the old generation of MELT G-C is the Gg-c heap). Generational copying GCs [11] handle quickly dead young temporary values by discarding them at once after having copied each live young value out of the birth region, but require a scan of all local variables, need to forward pointers to moved values, a write barrier, and normalization (like the administrative normal form in [7]) of explicit intermediate values inside calls ${ }^{19}$. This is awkward in hand-written $\mathrm{C}$ code but easy to generate. Minor MELT G-Cs are triggered before each call to gcc_collect (i.e. to the full Gg-c) to ensure that all live young MELT values have migrated to the old Gg-c heap. Compatibility between our MELT GC and Gg-c is thus achieved. An array of more than a hundred predefined values contains the only "global" MELT values (which are global roots for both the MELT GC and Gg-c).

MELT call frames are aggregated as local struct-ures, containing local MELT values, the currently called MELT closure, and local stuff (like raw tree pointers, etc.). Values inside these call frames are known to the MELT garbage collector, which scans them and possibly moves them. Expliciting these call frames facilitates introspective runtime reflection [18, 19, 20] at the MELT level; this might be useful for some future sophisticated analysis, e.g., in abstract interpretation [2, 3] of recursive functions, as a widening strategy. Concretely, local MELT values (and stuff) are aggregated in MELT call frames (represented as generated $C$ local struct-ures) organized in a single-linked list. This also enables the display of the MELT backtrace stack on errors.

\footnotetext{
${ }^{18}$ Ocaml bytecode contains both code and data; GNU \{ Emacs, CLisp, Smalltalk \} persist their entire heap image. But MELT has no persistent data files, to avoid serializing GCC's stuff (ie GCC's native data).

${ }^{19}$ That is, $f(g(x), y)$ should be normalized as $\tau=g(x) ; f(\tau, y)$ with $\tau$ being a fresh temporary.
} 


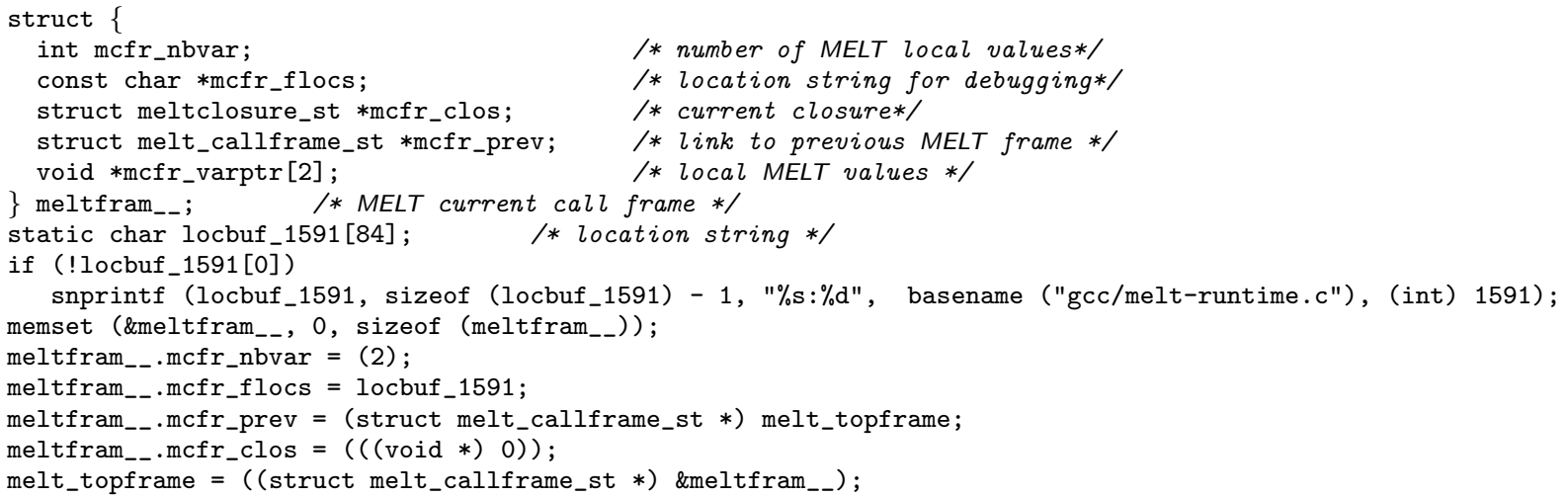

Figure 3: C preprocessor expansion of MELT_ENTERFRAME (2, NULL) at line 1591

The figure 2 gives an example of hand-written code following MELT conventions (a function meltgc_new_int boxing an integer into a value of given dicriminant and number to be boxed). It uses the MELT_ENTERFRAME macrd ${ }^{20}$, which is expanded by the $C$ preprocessor into the code in figure 3 , which declares and initialize the MELT call frame meltfram _. The MELT EXITFRAME () macro occurrence is expanded into melt_topframe $=$ (struct melt_callframe_st $*$ ) (meltfram (mcfr_prev) $_{\text {) }}$; to pop the current MELT frame. MELT provides a GCC pass checking some of MELT coding conventions in the hand-written part of the MELT runtime.

The MELT runtime depends deeply upon Gg-c, but does not depend much on the details of GCC's main data structures like e.g., tree or gimple or loop : our melt-runtime.c can usually be recompiled without changes when GCC's file gimple.h or tree.h changes, or when passes are changed or added in GCC's core. The MELT translator files warmelt*. melt (and the generated warmelt*.c files) don't depend really on GCC data structures like gimple. As a case in point, the major "gimple to tuple" transition 21 in gcc-4.4, which impacted a lot of GCC files, was smoothly handled within the MELT translator.

The MELT files which are actually processing GCC internal representations (like our xtramelt-*. melt or user MELT code), that is MELT code implementing new GCC passes, have to change only when the GCC API changes - exactly like other GCC passes. Often, since the change is compatible with existing code, these MELT files don't have to be changed at all (but should be recompiled into modules).

MELT handles two kinds of things: the first-class MELT values (allocated and managed in MELT's GC-ed heap) and other stuff, which are any other GCC data managed in C (either generated or handwritten C code within GCC $^{\text {melt }}$ ). Informally, Things $=$ Values $\cup$ Stuff. So raw long-s, edge-s or tree-s are stuff, and appear exactly in MELT memory like C-coded GCC passes handle them (without extra boxing). Variables and [sub-]expressions in MELT code, hence locals in MELT call frames, can be things of either kind (values or stuff).

Since $\mathrm{Gg}$-c requires each pointer to be of a gengtype- known type, values are really different from

\footnotetext{
${ }^{20}$ The Ocaml runtime has similar macros.

${ }^{21}$ In the old days of GCC version 4.3 the Gimple representation was physically implemented in tree-s and the C data structure gimple did not exist yet; at that time, Gimple was sharing the same physical structures as Trees and Generic [so Gimple was mostly a conventional restriction on Trees] - that is using many linked lists. The 4.4 release added the gimple structure to represent them, using arrays, not lists, for sibling nodes; this improved significantly GCC's performance but required patching many files.
} 
stuff. There is unfortunately no way to implement full polymorphism in MELT: we cannot have MELT tuples containing a mix of raw tree-s and MELT objects (even if both are Gg-c managed pointers). This Gg-c limitation has deep consequences in the MELT language (stuff, i.e. GCC native data, sadly cannot be first-class MELT values!).

Some parts of the MELT runtime are generated (by a special MELT mode). Various MELT values' and stuff implementation are described by MELT instances. So adding extra types of values, or interfacing additional GCC stuff to MELT, is fairly simple, but requires a complete re-building of MELT. Their GTY ( (...)) struct-ure declarations in $C$ are generated. Lower parts of the MELT runtime (allocating, forwarding, scanning routines - see chapters $6 \& 7$ of [11] - for the copying MELT G-C, hash-tables implementation, ...) are also generated. This generated $C$ code is kept in the source repository.

Notice that the distinction between first-class MELT values and plain stuff is essential in MELT, and is required by current GCC practices (notably its Gg-c collector). Therefore, the MELT language itself needs to denote them separately and explicitly, and the MELT runtime (and generated code) handles them differently. In that respect, MELT is not like Lisp, Scheme, Guile, Lua and Python. However, MELT coders should usually prefer handling values (the "first class citizens"), not raw stuff.

\subsection{MELT debugging aids}

When generating non-trivial $C$ code, it is important to lower the risk of crashing the generated code 22 This is achieved by systematically clearing all data (both values and raw stuff) to avoid uninitialized pointers (and MELT G-C also requires that), and by carefully coding low-level operations (primitives 3.4 .2 , c-matchers 4.3 , code chunks 3.4 .1 with tests against null pointers.

The generated $C$ code produced by the MELT translator contains many \#line directives (suitably wrapped with \#ifdef). In the rare cases when the gdb debugger needs to be used on MELT code (e.g., to deal with crashes or infinite loops), it will refer correctly to the originating MELT source file location. These positions are also written into MELT call frames, to ease backtracing on error.

MELT uses debug printing and assertions quite extensively. If enabled by the -fplugin-arg-melt-debug program argument to gcc, a lot of debug printing happens : each use of the debug_msg operation displays the current MELT source location, a message, and a value 23 For debugging stuff data, primitives debugtree, debuggimple, etc. are available. Assertions are provided by assert_msg which takes a message and a condition to check. When the check fails, the entire MELT call stack is printed (with positions referring to $*$.melt source files).

When variadic functions will be available in MELT, their first use will support polymorphic debug printing. A debug "macro" would be expanded into calls to a debug_at variading function, which would get the source location value as its first argument, and the values or stuff to be debug-printed as secondary variadic arguments.

An older version of MELT could be used with an external probe, which was a graphical program interacting with cc1 through asynchronous textual protocols. This approach required a quite invasive patch of GCC's code itself. The current GCC pass manager and plugin machinery now provides enough hooks, and future versions of MELT might communicate asynchronously with a central monitor (to be developed).

\footnotetext{
${ }^{22}$ However, it is still possible to make some MELT code crash, for instance by adding bugs in the $C$ form of our code chunks 3.4.1 In practice, MELT code crashes very rarely; most often it fails by breaking some assertions.

${ }^{23}$ Values are printed for debug use with MELT message passing through the DBG_OUTPUT \& DBG_OUTPUTAGAIN selectors.
} 


\section{The MELT language and its pecularities}

Some familiarity with a Lisp-like language (like Emacs Lisp, Scheme, Common Lisp, etc.) is welcome to understand this section. Acquaintance with a dynamically typed scripting language like Python, Guile or Ruby could also help. See the web site gcc-melt. org for more material (notably tutorials) on MELT.

MELT has a Lisp-like syntax because it was (at its very beginning) implemented with an initial "external" MELT to C translator prototyped in Common Lisp. Since then, a lot of newer features have been progressively added (using an older version of MELT to bootstrap its current version). The Emacs Lisp language (in the Emacs editor), Guile (the Gnu implementation of Scheme), and machine description files in GCC back-end are successful examples of other Lisp dialects within Gnu software. Finally, existing editing modes ${ }^{24}$ for Lisp are sufficient for MELT.

An alternative infix syntax (code-named Milt) for MELT is in the works; the idea is to have an infix parser, coded in MELT, for future *.milt files, which is parsed into MELT internal s-expressions (i.e. into the same instances of CLASS_SEXPR as the MELT Lisp-like reader does): symbols starting with + or - are parsed as infix operators (like Ocaml does) with additive precedences, those starting with $*$ or / have multiplicative precedence, etc.

MELT shares with existing Lisp languages many syntactic and lexical conventions for comments, indentation, symbols (which may be non alpha-numerical), case-insensitivity, and a lot of syntax (like if, let, letrec, defun, cond ...). As in all Lisp dialects, everything is parenthesized like ( operator operands ... ) so parenthesis are highly significant. The quote, back-quote, comma and question mark characters have special significance, so ' $a$ is parsed exactly as (quote $a$ ), ?b as (question b) etc. Like in Common Lisp, words prefixed with a colon like : long are considered as "keywords" and are not subject to evaluation. Symbols and keywords exist both in source files and in the running MELT heap.

\subsection{MELT macro-strings}

Since "mixing" $C$ code chunks ( 3.4 .1 inside MELT code is very important, simple meta-programming is implemented by a lexical trick 25 ; macro-strings are strings prefixed with \#\{ and suffixed with $\} \#$ and are parsed specially; these prefix and suffix strings have been chosen because they usually don't appear in $C$ code. Within a macro-string, backslash does not escape characters, but $\$$ and sometimes \# are scanned specially, to parse symbols inside macro-strings.

For example, MELT reads the macro-string $\quad \#\{/ * \$ P \# A * / \operatorname{printf}(" \mathrm{a}=\% 1 \mathrm{~d} \backslash \mathrm{n} ", \$ \mathrm{~A}) ;\} \# \quad$ exactly as a list $(" / * " \quad p \quad$ "A*/printf $(\backslash " a=\% l d \backslash \backslash n \backslash ", "$ a " $) ; ") \quad$ of 5 elements whose $1^{\text {st }}$, $3^{\text {rd }}$ and $5^{\text {th }}$ elements are string ${ }^{26}$ and $2^{\text {nd }}$ and $4^{\text {th }}$ elements are symbols $\mathrm{p}$ and $\mathrm{a}$. This is useful when one wants to mix C code inside MELT code; some macro-strings are several dozens of lines long, but don't need any extra escapes (as would be required by using plain strings).

Another example of macro-string is given in the following "hello-world" (complete) MELT program:

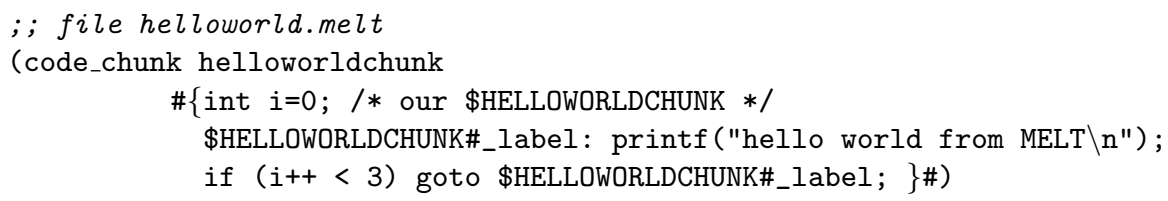

\footnotetext{
${ }^{24}$ Emacs mode for Lisp is nearly enough for editing, highlighting and indenting MELT code.

${ }^{25}$ Inspired by handling of $\$$ in strings or "here-documents" by shells, Perl, Ruby, ...

${ }^{26}$ The first string has the two characters /* and the last has the two characters );
} 
The macro-string spans on 3 lines, and contains some $C$ code with the helloworldchunk MELT symbol. The above helloworld.melt file (of 4 lines) is translated into a helloworld.c file (of 389 lines ${ }^{27}$ in $C$ ). It uses the code_chunk construct explained in $\$ 3.4 .1$ below (to emit translated $C$ code).

\subsection{MELT values and stuff}

Every MELT value has a discriminant (at the start of the memory zone containing that value). As an exception, nil ${ }^{28}$, represented by the $\mathrm{C}$ null pointer has conventionally a specific discriminant DISCR_NULL_RECIEVER. The discriminant of a value is used by the MELT runtime, by Gg-C and in MELT code to separate them. MELT values can be boxed stuff (e.g., boxed long or boxed tree), closures, lists, pairs, tuples, boxed strings, ..., and MELT objects. Several predefined objects, e.g., CLASS_CLASS, DISCR_NULL_RECEIVER . .., are required by the MELT runtime. The hierarchy of discriminants is rooted at DISCR_ANY_RECEIVER 29. Discriminants are objects (of CLASS_DISCRIMINANT). Core classes and discriminants are predefined as MELT values (known by both Gg-c and MELT G-C).

Each MELT object has its class as its discriminant. Classes are themselves objects and are organized in a single-inheritance hierarchy rooted at CLASS_ROOT (whose parent discriminant is DISCR_ANY_RECIEVER). Objects are represented in C as exactly a structure with its class (i.e. discriminant) obj_class, its unsigned hash-code obj_hash (initialized once and for all), an unsigned "magic" short number obj_num, the unsigned short number of fields obj_len, and the obj_vartab [obj_len] array of fields, which are MELT values. The obj_num in objects can be set at most once to a non-zero unsigned short, and may be used as a tag: MELT and Gg-c discriminate quickly a value's data-type (for marking, scanning and other purposes) through the obj_num of their discriminant. So, safely testing in $C$ if a value $\mathrm{p}$ is a MELT closure is as fast as $\mathrm{p} !=$ NULL \&\& $\mathrm{p}->$ discr->obj_num $==$ MELTOBMAG_CLOSURE.

MELT field descriptors and method selectors are objects. Every MELT value (object or not, even nil) can be sent a message, since its discriminant (i.e., its class, if it is an object) has a method map (a hash table associating selectors to method bodies) and a parent discriminant (or super-class). Message passing in MELT is similar to those in Smalltalk and Ruby. Method bodies can be dynamically installed with (install_method discriminant selector function) and removed at any time in any discriminant or class. Method invocations use the method hash-maps (similar to methods' dictionnaries in Smalltalk) to find the actual method to run.

The MELT reader produces mostly objects and sometimes other values: S-expressions are parsed as instances of CLASS_SEXPR (containing the expression's source location and the list of its components); symbols (like $==$ or let or $\mathrm{x}$ ) as instances of CLASS_SYMBOL; keywords like $:$ long or : else as instances of CLASS_KEYWORD; numbers like -1 as values of DISCR_INTEGER etc.

Each stuff (that is, non-value things like long or tree ...) have its boxed value counterpart, so boxed gimple-s are values containing, in addition of their discriminant (like DISCR_GIMPLE), a raw gimple pointer.

In MELT expressions, literal integers like 23 or strings like "hello $\backslash \mathrm{n}$ " refer to raw :long or : cstring stuff ${ }^{30}$, not constant values. To be considered as MELT values they need to be quoted, so (contrarily to other Lisps) in MELT $2 \not \equiv ' 2$ : the plain 2 denotes a raw stuff of c-type :long so is not a value, but the

\footnotetext{
${ }^{27}$ With 260 lines of code, including 111 preprocessor directives, mostly \#line, and 129 comment or blank lines, and all the code doing "initialization".

${ }^{28}$ As in Common Lisp or Emacs Lisp (or C itself), but not as in Scheme, MELT nil value is considered as false, and every non-nil value is true.

${ }^{29}$ DISCR_ANY_RECEIVER is rarely used, e.g., to install catch-all method handlers.

${ }^{30}$ All : cstring are (const char*) C-strings in the text segment of the executable, so they are not malloc-ed.
} 
quoted expression ' 2 denotes the boxed integer 2 constant value of DISCR_CONSTANT_INTEGER so they are not equivalent! As in Lisp, a quoted symbol like ' $j$ denotes a constant value (of CLASS_SYMBOL).

To associate things (either MELT objects or GCC stuff, all of the same type) to MELT values, hashmaps are extensively used: so homogenous hash tables keyed by objects, raw strings, or raw stuff like tree-s or gimple-s ... are values (of discriminant DISCR_MAP_OBJECTS ..., DISCR_MAP_TREES). While hash-maps are more costly than direct fields in structures to associate some data to these structures, they have the important benefit of avoiding disturbing existing data structures of GCC. And even C plugins of GCC cannot add for their own convenience extra fields into the carefully tuned tree or gimple structures of GCC's tree.h or gimple.h.

Aggregate MELT values include not only objects, hash-tables and pairs, but also tuples (a value containing a fixed number of immutable component values), closures, lists, ... Lists know their first and last pairs. Aggregate values of the same kind may have various discriminants. For instance, within a MELT class (which is itself a MELT object of CLASS_CLASS) a field gives the tuple of all super-classes starting with CLASS_ROOT. That tuple has DISCR_CLASS_SEQUENCE as discriminant, while most other tuples have DISCR_MULT IPLE as discriminant.

Decaying values may help algorithms using memoization; they contain a value reference and a counter, decremented at each major garbage collection. When the counter reaches 0 , the reference is cleared to nil.

Adding a new important GCC C type like gimple ${ }^{31}$ for some new stuff is fairly simple: add (in MELT code) a new predefined C-type descriptor (like CTYPE_G IMPLE referring to keyword :gimple) and additional discriminants, and regenerate all of MELT. C-type descriptors (e.g., CTYPE_EDGE) and value type descriptors (like VALDESC_LIST) contains dozen[s] of fields (names or body chunk of generated $C$ routines) used when generating the runtime support routines.

The :void keyword (and so CTYPE_VOID) is used for side-effecting code without results. C-type keywords (like :void, :long, :tree, :value, :gimple, :gimple_seq, etc.) qualify (in MELT source code) formal arguments, local variables (bound by let, ...), etc.

MELT is typed for things: e.g., the translator complains if the +i primitive addition operator (expecting two raw : long stuff and giving a : long result) is given a value or a :tree argument. Furthermore, let bindings can be explicitly typed (by default they bind a value). Within values, typing is dynamic; for instance, a value is checked at runtime to be a closure before being applied. When applying a MELT closure to arguments, the first argument, if any, needs to be a value (it would be the receiver if the closure is a method for message passing ${ }^{32}$, others can be things, i.e. values or stuff. In MELT applications, the types of secondary arguments and secondary results are described by constant byte strings, and the secondary arguments or results are passed (in generated $C$ code) as an array of unions. The generated MELT function prologue (in $C$ ) checks that the formal and actual type of secondary arguments are the same (otherwise, argument passing stops, and all following actual arguments are cleared).

All MELT things (value or stuff), in particular local variables (or mismatched formals), are initially cleared (usually by zeroing the whole MELT call frame in the $C$ prologue of each generated routine). So MELT values are initially () (i.e., nil in MELT syntax), a : tree stuff is initially the null tree (i.e. (tree) 0 in $C$ syntax), a :long stuff is initially OL, a :cstring stuff is initialized to (const char*)0. Notice that cleared stuff is considered as false in conditional context.

\footnotetext{
${ }^{31}$ This kind of radical addition don't happen often in the GCC community because it usually impacts a lot of GCC files.

${ }^{32}$ The somehow arbitrary requirement of having the first argument of every MELT function be a value speeds up calls to functions with one single value argument, and permits using closures as methods without checks: sending a message to a raw stuff like e.g., a tree won't work.
} 
Functions written in MELT (with defun for named functions or lambda for anonymous ones) always return a value as their primary result (which may be ignored by the caller, and defaults to nil). The first formal argument (if any) and the primary result of MELT functions should be values (so nested function calls deal mainly with values). Secondary arguments and results can be any things (each one is either a value or some stuff). The (multicall ...) syntax binds primary and secondary results like Common Lisp's multiple-value-bind.

\subsection{Syntax overview}

The following constructs should be familiar (except the last one, match, for pattern matching) since they look like in other Lisps. Notice that our let is always sequentia 33 . Formals in abstractions 34 are restricted to start with a formal value; this speeds up the common case of functions with a single value argument, and facilitates installation of any function as method (without checking that the formal reciever is indeed a value).

List of formal arguments (in lambda, defun etc.) contains either symbols (which are names of formals bound by e.g., the lambda) like $\mathrm{x}$ or discr, or c-type keywords like :value or :long or :gimple .... A c-type keyword qualify all successing formals up to the next c-type keywords, and the default c-type is : value. For example, the formal arguments list ( $\mathrm{x}$ y :long $\mathrm{n} \mathrm{k}$ : gimple $\mathrm{g}$ : value $\mathrm{v}$ ) have 6 formals : $\mathrm{x} \mathrm{y} \mathrm{v}$ are MELT values, $\mathrm{n} \mathrm{k}$ are raw long stuff, $\mathrm{g}$ is a raw gimple stuff.

Local bindings (in let or letrec) has an optional c-type annotation, then the newly bound symbol, then the sub-expression bounding it. So (:long $x$ 2) locally binds (in the body of the enclosing let) the symbol $\mathrm{x}$ to the raw long stuff 2 , and in the let body $\mathrm{x}$ is a raw long variable.

Patterns and pattern matching are explained in $\$ 4$

\section{expressions where $n \geq 0$ and $p \geq 0$}

\begin{tabular}{|c|c|c|}
\hline application & $\left(\begin{array}{llll}\phi & \alpha_{1} & \ldots & \alpha_{n}\end{array}\right)$ & apply function (or primitive) $\phi$ to arguments $\alpha_{i}$ \\
\hline assignment & $(\operatorname{setq} v \varepsilon)$ & set local variable $v$ to $\varepsilon$ \\
\hline message passing & $\left(\begin{array}{lllll}\sigma & \rho & \alpha_{1} & \ldots & \alpha_{n}\end{array}\right)$ & send selector $\sigma$ to reciever $\rho$ with arguments $\alpha_{i}$ \\
\hline let expression & (let $\left.\left(\beta_{1} \ldots \beta_{n}\right) \quad \varepsilon_{1} \ldots \varepsilon_{p} \varepsilon^{\prime}\right)$ & $\begin{array}{l}\text { with local sequential bindings } \beta_{i} \text { evaluate side- } \\
\text { effecting sub-expressions } \varepsilon_{j} \text { and give result of } \varepsilon^{\prime}\end{array}$ \\
\hline sequence & $\left(\right.$ progn $\left.\varepsilon_{1} \ldots \varepsilon_{n} \varepsilon^{\prime}\right)$ & $\begin{array}{l}\text { evaluate } \varepsilon_{i} \text { (for their side effects) and at last } \varepsilon^{\prime} \text {, giving its } \\
\text { result (like the operator, in } \mathrm{C} \text { ) }\end{array}$ \\
\hline abstraction & (lambda $\left.\phi \quad \varepsilon_{1} \ldots \varepsilon_{n} \varepsilon^{\prime}\right)$ & $\begin{array}{l}\text { anonymous function with formals } \phi \text { and side- } \\
\text { effecting expressions } \varepsilon_{i} \text {, return result of } \varepsilon^{\prime}\end{array}$ \\
\hline pattern matchin & $\left(\begin{array}{lllll}\text { match } & \varepsilon & \chi_{1} & \ldots & \chi_{n}\end{array}\right)$ & $\begin{array}{l}\text { match result of } \varepsilon \text { against match clauses } \chi_{i} \text {, giving } \\
\text { result of last expression of matched clause. }\end{array}$ \\
\hline
\end{tabular}

Conditional expressions alter control flow as usual. However, conditions can be things, e.g., the 0 : long stuff is false, other long stuff are true, a gimple stuff is false iff it is the null gimple pointer, etc. The "else" part $\varepsilon$ of an if test is optional. When missing, it is false, that is a cleared thing. Notice that tested conditions and the result of a conditional expression can be either values or raw stuff, but all the conditional sub-expressions of a condition should have consistent types, otherwise the entire expression has : void type.

\footnotetext{
${ }^{33}$ So the let of MELT is like the let* of Scheme!

${ }^{34}$ Notice that lambda abstractions are constructive expressions and may appear in letrec or let bindings.
} 
conditional expressions where $n \geq 0$ and $p \geq 0$

\begin{tabular}{l|l|l}
\hline test & $\left(\begin{array}{llll}\text { if } \tau & \theta & \varepsilon\end{array}\right)$ & if $\tau$ then $\theta$ else $\varepsilon$ (like ?: in C) \\
\hline conditional & $\left(\begin{array}{lllll}\text { cond } \kappa_{1} & \ldots & \kappa_{n}\end{array}\right)$ & evaluate conditions $\kappa_{i}$ until one is satisfied \\
\hline conjunction & $\left.\begin{array}{lllll}\text { and } \kappa_{1} & \ldots & \kappa_{n} & \kappa^{\prime}\end{array}\right)$ & $\begin{array}{l}\text { if } \kappa_{1} \text { and then } \kappa_{2} \ldots \text { and then } \kappa_{n} \text { is "true" (non nil or non zero) then } \\
\kappa^{\prime} \text { otherwise the cleared thing of same type }\end{array}$ \\
\hline disjunction & $\left.\begin{array}{lllll}\text { or } \delta_{1} & \ldots & \delta_{n}\end{array}\right)$ & $\begin{array}{l}\delta_{1} \text { or else } \delta_{2} \ldots=\text { the first of the } \delta_{i} \text { which is "true" (non nil, or non } \\
\text { zero, } \ldots\end{array}$
\end{tabular}

In a cond expression, every condition $\kappa_{i}$ (except perhaps the last) is like $\left(\begin{array}{lllll}\gamma_{i} & \varepsilon_{i, 1} & \ldots & \varepsilon_{i, p_{i}} & \varepsilon^{\prime}\end{array}\right)$ with $p_{i} \geq 0$. The first such condition for which $\gamma_{i}$ is "true" gets its sub-expressions $\varepsilon_{i, j}$ evaluated sequentially for their side-effects and gives the result of $\varepsilon^{\prime}$. The last condition can be (:else $\varepsilon_{1} \ldots \varepsilon_{n} \varepsilon^{\prime}$ ), is triggered if all previous conditions failed, and (with the sub-expressions $\varepsilon_{i}$ evaluated sequentially for their side-effects) gives the result of $\varepsilon^{\prime}$

MELT has some more expressions.

more expressions

\begin{tabular}{|c|c|c|}
\hline loop & (forever $\begin{array}{llll}\lambda & \alpha_{1} & \ldots & \alpha_{n} \text { ) }\end{array}$ & $\begin{array}{l}\text { loop indefinitely on the } \alpha_{i} \text { which may } \\
\text { exit }\end{array}$ \\
\hline exit & $\left(\begin{array}{llllll}\text { (exit } \lambda & \varepsilon_{1} & \ldots & \varepsilon_{n} & \left.\varepsilon^{\prime}\right)\end{array}\right.$ & $\begin{array}{l}\text { exit enclosing loop } \lambda \text { after side-effects } \\
\text { of } \varepsilon_{i} \text { and result of } \varepsilon^{\prime}\end{array}$ \\
\hline return & $\left(\begin{array}{lllll}\text { return } & \varepsilon & \varepsilon_{1} & \ldots & \left.\varepsilon_{n}\right)\end{array}\right.$ & $\begin{array}{l}\text { return } \varepsilon \text { as the main result, and the } \varepsilon_{i} \text { as } \\
\text { secondary results }\end{array}$ \\
\hline multiple call & (multicall $\left.\phi \quad \kappa \quad \varepsilon_{1} \ldots \varepsilon_{n} \varepsilon^{\prime}\right)$ & $\begin{array}{l}\text { locally bind formals } \phi \text { to main and sec- } \\
\text { ondary result[s] of application or send } \kappa \\
\text { and evaluate the } \varepsilon_{i} \text { for side-effects and } \\
\varepsilon^{\prime} \text { for result }\end{array}$ \\
\hline recursive let & (letrec $\left.\left(\beta_{1} \ldots \beta_{n}\right) \varepsilon_{1} \ldots \varepsilon_{p}\right)$ & $\begin{array}{l}\text { with [mutually-] recursive constructive } \\
\text { bindings } \beta_{i} \text { evaluate sub-expressions } \varepsilon_{j}\end{array}$ \\
\hline field access & (get_field : $\Phi \varepsilon$ ) & $\begin{array}{l}\text { if } \varepsilon \text { gives an appropriate object retrieves } \\
\text { its field } \Phi \text {, otherwise nil }\end{array}$ \\
\hline unsafe field access & (unsafe_get_field : $\Phi \varepsilon$ ) & $\begin{array}{l}\text { unsafe access without check like the } \\
\text { above operation }\end{array}$ \\
\hline object update & (put_fields $\left.\varepsilon \quad: \Phi_{1} \quad \varepsilon_{1} \quad \ldots \quad: \Phi_{n} \quad \varepsilon_{n}\right)$ & $\begin{array}{l}\text { safely update (if appropriate) in the ob- } \\
\text { ject given by } \varepsilon \text { each field } \Phi_{i} \text { with } \varepsilon_{i}\end{array}$ \\
\hline unsafe object update & (unsafe_put_fields $\varepsilon: \Phi_{1} \varepsilon_{1} \quad \ldots$ ) & unsafely update the object given by $\varepsilon$ \\
\hline
\end{tabular}

The unsafe field access unsafe_get_field is reserved to expert MELT programmers, since it may crash. The safer variant test that the expression $\varepsilon$ evaluates ${ }^{35}$ to a MELT object of appropriate class before accessing a field $\Phi$ in it. Field updates with put_fields are safe ${ }^{36}$, with an unsafe but quicker variant unsafe_put_fields available for MELT experts.

Mutually recursive letrec bindings should have only constructive expressions. constructive expressions

\begin{tabular}{l|llllll}
\hline list & $\left(\begin{array}{llllll}\text { list } \alpha_{1} & \ldots & \alpha_{n}\end{array}\right)$ & & make a list of $n$ values $\alpha_{i}$ \\
\hline tuple & $\left(\begin{array}{lllllll}\text { tuple } \alpha_{1} & \ldots & \alpha_{n}\end{array}\right)$ & & make a tuple of $n$ values $\alpha_{i}$ \\
\hline instance & $\left.\begin{array}{lllllll}\text { (instance } & \kappa & : \Phi_{1} & \varepsilon_{1} & \ldots & : \Phi_{n} & \varepsilon_{n}\end{array}\right)$ & $\begin{array}{l}\text { make an instance of class } \kappa \text { and } n \\
\text { fields } \Phi_{i} \text { set to value } \varepsilon_{i}\end{array}$
\end{tabular}

\footnotetext{
${ }^{35}$ I.e. test if the value $\omega$ of $\varepsilon$ is an object which is a direct or indirect instance of the class defining field $\Phi$, otherwise a nil value is given.

${ }^{36}$ Update object $\omega$, value of $\varepsilon$, only if it is an object which is a direct or indirect instance of the class defining each field $\Phi_{i}$
} 
Of course lambda expressions are also constructive and can appear inside letrec. Notice that since MELT is translated into $\mathrm{C}$, and because of runtime constraints, MELT recursion is never handled tailrecursively so always consume stack space. This also motivates iterative constructions (like forever and our iterators).

Name defining expressions have a syntax starting with def. Most of them (except defun, defclass, definstance) have no equivalent in other languages, because they define bindings related to $\mathrm{C}$ code generation. For the MELT translator, bindings have various kinds; each binding kind is implemented as some subclass of CLASS_ANY_BINDING.

Name exporting expressions are essentially directives for the module system of MELT. Only exported names are visible outside a module. A module initialization expects a parent environment and produces a newer environment containing exported bindings. Both name defining and exporting expressions are supposed to appear only at the top-level (and should not be nested inside other MELT expressions).

\begin{tabular}{|c|c|c|}
\hline \multicolumn{3}{|c|}{ expressions defining names } \\
\hline for functions & $\left(\right.$ defun $\left.v \begin{array}{lllll}v & \varepsilon_{1} & \ldots & \varepsilon_{n} & \varepsilon^{\prime}\end{array}\right)$ & $\begin{array}{l}\text { define function } v \text { with formal arguments } \phi \text { and body } \varepsilon_{1} \\
\ldots \varepsilon_{n} \varepsilon^{\prime}\end{array}$ \\
\hline for classes & $\begin{array}{l}(\text { defclass } v \text { :super } \sigma \text { :fields } \\
\left(\phi_{1} \ldots \phi_{n}\right) \text { ) }\end{array}$ & define class $v$ of super-class $\sigma$ and own fields $\phi_{i}$ \\
\hline for instances & $\begin{array}{lllllllll}\text { (definstance } & l & \kappa & : f_{1} & \varepsilon_{1} & \ldots & : f_{n} & \left.\varepsilon_{n}\right)\end{array}$ & $\begin{array}{l}\text { define an instance } l \text { of class } \kappa \text { with each field } f_{i} \\
\text { initialized to the value of } \varepsilon_{i}\end{array}$ \\
\hline for selectors & $\begin{array}{l}\text { (defselector } \sigma \kappa[\text { :formals } \Psi]: f_{1} \\
\left.\varepsilon_{1} \quad \ldots \quad: f_{n} \varepsilon_{n}\right)\end{array}$ & $\begin{array}{l}\text { define an selector } l \text { of class } \kappa \\
\text { CLASS_SELECTOR) with each extra field } f_{i} \\
\text { initialized to the value of } \varepsilon_{i} \text { (usually no extra fields } \\
\text { are given so } n=0 \text { ) and with optional formals } \Psi\end{array}$ \\
\hline for primitives & (defprimitive $\left.\begin{array}{llll} & \phi & : \theta & \eta\end{array}\right)$ & $\begin{array}{l}\text { define primitive } v \text { with formal arguments } \phi \text {, result c-type } \\
\theta \text { by macro-string expansion } \eta\end{array}$ \\
\hline for c-iterators & (defciterator $v \Phi \Phi \quad \sigma \quad \Psi \quad \eta \eta^{\prime}$ ) & $\begin{array}{l}\text { define c-iterator } v \text { with input formals } \Phi \text {, state symbol } \\
\sigma \text {, local formals } \Psi \text {, start expansion } \eta \text {, end expansion } \eta^{\prime}\end{array}$ \\
\hline for c-matchers & (defcmatcher $v \quad \Phi \Psi \Psi \quad \sigma \quad \eta \quad \eta^{\prime}$ ) & $\begin{array}{l}\text { define c-matcher } v \text { with input formals } \Phi \text { [the matched } \\
\text { thing, then other inputs], output formals } \Psi \text {, state symbol } \\
\sigma \text {, test expansion } \eta \text {, fill expansion } \eta^{\prime}\end{array}$ \\
\hline for fun-matchers & (defunmatcher $v \Phi \Psi \varepsilon$ ) & $\begin{array}{l}\text { define funmatcher } v \text { with input formals } \Phi \text {, output for- } \\
\text { mals } \Psi \text {, with function } \varepsilon\end{array}$ \\
\hline
\end{tabular}

\begin{tabular}{|c|c|c|}
\hline \multicolumn{3}{|c|}{ expressions exporting names } \\
\hline of values & (export_value $v_{1} \quad \ldots$ ) & $\begin{array}{l}\text { export the names } v_{i} \text { as bindings of values (e.g., of } \\
\text { functions, objects, matcher, selector, ...) }\end{array}$ \\
\hline of macros & (export_macro $v \varepsilon$ ) & $\begin{array}{l}\text { export name } v \text { as a binding of a macro (expanded by } \\
\text { the } \varepsilon \text { function) }\end{array}$ \\
\hline of classes & (export_class $\left.v_{1} \ldots\right)$ & $\begin{array}{l}\text { export every class name } v_{i} \text { and all their own fields } \\
\text { (as value bindings) }\end{array}$ \\
\hline as synonym & (export_synonym $v v^{\prime}$ ) & $\begin{array}{l}\text { export the new name } v \text { as a synonym of the existing } \\
\text { name } v^{\prime}\end{array}$ \\
\hline
\end{tabular}

Macro-expansion is internally the first step of MELT translation to C: parsed (or in-heap) S-exprs (of CLASS_SEXPR) are macro-expanded into a MELT "abstract syntax tree" (a subclass of CLASS_SOURCE). This macro machinery is extensively used, e.g., let and if constructs are macro-expanded (to instances of CLASS_SOURCE_LET Or CLASS_SOURCE_IF respectively.

Field names and class names are supposed to be globally unique, to enable checking their access or update. Conventionally class names start with CLASS - and field names usually share a common unique prefix in their class. There is no protection (i.e. visibility restriction like private in $\mathrm{C}++$ ) for accessing a field. 
All definitions accept documentation annotation using : doc, and a documentation generator mode produces documentation with-cross references in Texinfo format.

Miscellanous constructs are available, to help in debugging or coding or to generate various $\mathrm{C}$ code depending on compile-time conditions.

\begin{tabular}{|c|c|c|}
\hline \multicolumn{3}{|r|}{ expressions for debugging } \\
\hline assert check & (assert_msg $\mu \tau$ ) & nice "halt" showing message $\mu$ when asserted test $\tau$ is false \\
\hline warning & (compile_warning $\mu \varepsilon$ ) & $\begin{array}{l}\text { like \#warning in C: emit warning } \mu \text { at MELT translation time and gives } \\
\varepsilon\end{array}$ \\
\hline \multicolumn{3}{|r|}{ meta-conditionals } \\
\hline Cpp test & $\left(\right.$ cppif $\left.\sigma \varepsilon \varepsilon \varepsilon^{\prime}\right)$ & conditional on a preprocessor symbol: emitted $C$ code is \#if $\sigma$ code for $\varepsilon$ \\
\hline & & $\#$ \#lse code for $\varepsilon^{\prime} \#$ endif \\
\hline
\end{tabular}

Reflective access to the current and parent environment is possible (but useful in exceptional cases, since export_... directives are available to extend the current exported environment):

\begin{tabular}{|c|c|c|}
\hline & essions & \\
\hline Parent environment & (parent_module_environment) & gives the previous module environment \\
\hline Current environment & (current_module_environment_container) & $\begin{array}{l}\text { gives the container of the current module's } \\
\text { environment }\end{array}$ \\
\hline
\end{tabular}

\subsection{Linguistic constructs to fit MELT into GCC}

Several language constructs are available to help fit MELT into GCC, taking advantage of MELT and GCC runtime infrastructure (notably $\mathrm{Gg}-\mathrm{c}$ ). They usually use macro-strings to provide $\mathrm{C}$ code with holes. Code chunks (3.4.1) simply permit to insert $\mathrm{C}$ code in MELT code. Higher-level constructs describe how to translate other MELT expressions into C: primitives (3.4.2) describe how to translate low-level operations into C; c-iterators ( $\$ 3.4 .3$ define how iterative expressions are translated into for-like loops; c-matchers ( 4.3 define how to generate simple patterns (for matching), etc.

\subsubsection{Code chunks}

Code chunks are simple MELT templates (of :void c-type) for generated $C$ code. They are the lowest possible way of impacting MELT $C$ code generation, so are seldom used in MELT (like asm is rarely used in $C$ ).

As a trivial example where $i$ is a MELT: long variable bound in an enclosing let,

(code_chunk sta

$\#\{$ \$sta\#_lab: printf("i=\%ld $\backslash n ", \$ i++) ;$ goto $\$$ sta\#_lab; $\} \#)$

would be translated to

\{sta_1_lab: printf("i=\%ld $\backslash n "$, curfnum[3]++); goto sta_1_lab;

the first time it translated (i becoming curfnum [3] in C), but would use sta_2_lab the second time, etc. The first argument of code_chunk - sta here - is a state symbol, expanded to a $\mathrm{C}$ identifier unique to the code chunk's translation. The second argument is the macro-string serving as template to the generated $\mathrm{C}$ code. The state symbol is uniquely expanded, and other symbols should be MELT variables and are replaced by their translation. So the code_chunk of state symbol helloworldchunk in $\$ 3.1$ is translated into the following $C$ code:

int $i=0 ; / *$ our HELLOWORLDCHUNK

HELLOWORLDCHUNK__1_label: printf("hello world from MELT $\backslash \mathrm{n}$ ") ;

if $(i++<3$ ) goto HELLOWORLDCHUNK__1_label; ; 


\subsubsection{Primitives}

Primitives define a MELT operator by its C expansion. The unary negation negi is defined exactly as :

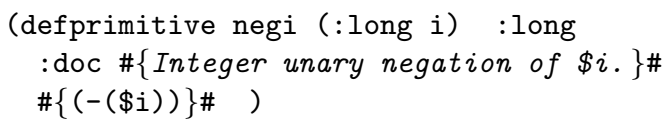

Here we specify that the formal argument $i$ is, like the result of negi, a :long stuff. We give an optional documentation, followed by the macro-string for the $\mathrm{C}$ expansion. Primitives don't have state variables but are subject to normalization ${ }^{37}$ and type checking. During expansion, the formals appearing in the primitive definition are replaced appropriately.

\subsubsection{C-iterators}

A MELT c-iterator is an operator translated into a for-like C loop. The GCC compiler defines many constructs similar to $\mathrm{C}$ for loops, usually with a mixture of macros and/or trivial inlined functions. $\mathrm{C}$-iterators are needed in MELT because the GCC API defines many iterative conventions. For example, to iterate on every gimple $g$ inside a given gimple_seq $s$ GCC mandates (see $\$ 1.1$ ) the use of a gimple_simple_iterator.

In MELT, to iterate on the :gimpleseq s obtained by the expression $\sigma$ and do something on every :gimple g inside s, we can simply code (let ( (:gimpleseq s $\sigma$ ) ) (each_in_gimpleseq (s) (:gimple g) 「do something with g....)) by invoking the $c$-iterator each_in_gimpleseq, with a list of inputs - here simply (s) - and a list of local formals - here (:gimple g) - as the iterated things.

This c-iterator (a template for such for-like loops) is defined exactly as:

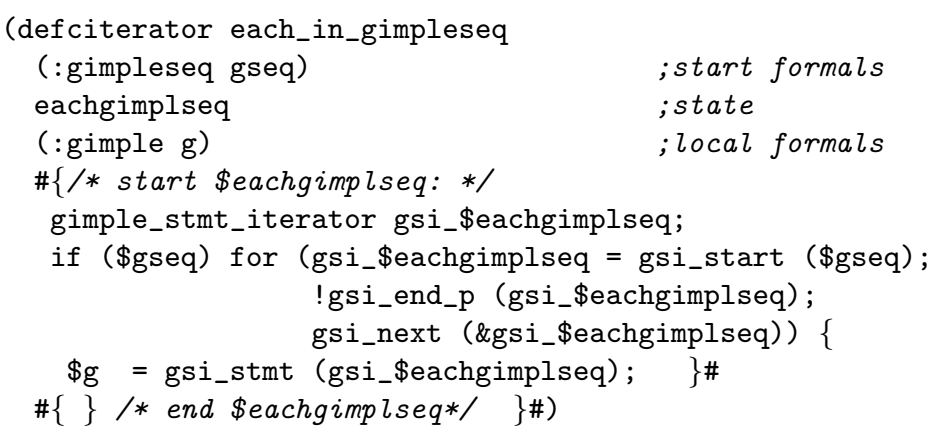

We give the start formals, state symbol, local formals and the "before" and "after" expansion of the generated loop block. The expansion of the body of the invocation goes between the before and after expansions. C-iterator occurrences are also normalized (like primitive occurrences are). MELT expressions using c-iterators give a :void result, since they are used only for their side effects.

\subsection{Modules, environments, standard library and hooks}

A single $*$. melt source file ${ }^{38}$ is translated into a single module loaded by the MELT run-time. The module's generated start_module melt routine [often quite big] takes a parent environment, executes the top-level forms, and finally returns the newly created module's environment. Environments and their bindings are reified as objects.

\footnotetext{
${ }^{37}$ Assuming that $\mathrm{x}$ is a MELT variable for a : long stuff, then the expression ( $+\mathrm{i}$ (negi $\mathrm{x}$ ) 1) is normalized as let $\alpha=$ $-x, \beta=\alpha+1$ in $\beta$ in pseudo-code - suitably represented inside MELT (where $\alpha, \beta$ are fresh gensym-ed variables).

${ }^{38} \mathrm{MELT}$ can also translate into $\mathrm{C}$ a sequence of S-expressions from memory, and then dynamically load the corresponding temporary module after it has been $\mathrm{C}$-compiled.
} 
Only exported names add bindings in the module's environment. MELT code can explicitly export defined values (like instances, selectors, functions, c-matchers, ...) using the (export_values ...) construct; macros (or pat-macros [that is pattern-macros producing abstract syntax of patterns]) definitions are exported using the (export_macro ...) construct or (export_patmacro ...); classes and their own fields are exported using the (export_class ...) construct. Macros and pattern macros in MELT are expanded into an abstract syntax tree (made of objects of sub-classes of CLASS_SOURCE, e.g., instances of CLASS_SOURCE_LET or of CLASS_SOURCE_APPLY, ...), not into s-expressions (i.e. objects of CLASS_SEXPR, as provided by the reader).

Field names should be globally unique: this enables (get_field :named_name $\mathrm{x}$ ) to be safely translated into something like "if $\mathrm{x}$ is an instance of CLASS_NAMED fetch its : named name field otherwise give nil", since MELT knows that named_name is a field of CLASS_NAMED.

As in C, there is only one name-space in MELT which is technically, like Scheme, a Lisp ${ }_{1}$ dialec 39 (in Queinnec's terminology [22]). This prompts a few naming conventions: most exported names of a module share a common prefix; most field names of a given class share the same prefix unique to the class, etc.

The entire MELT translation process [26] is implemented through many exported definitions which can be used by expert MELT users to customize the MELT language to suit their needs. Language constructs ${ }^{40}$ give total access to environments (instances of CLASS_ENVIRONMENT).

Hooks for changing GCC's behavior are provided on top of the existing GCC plugin hooks (for instance, as exported primitives like install_melt_gcc_pass which installs a MELT instance describing a GCC pass and registers it inside GCC).

A fairly extensive MELT standard library is available (and is used by the MELT translator), providing many common facilities (map-reduce operations; debug output methods; run-time asserts printing the MELT call stack on failure; translate-time conditionals emitted as \#ifdef; ...) and interfaces to GCC internals. Its .texi documentation is produced by a generator inside the MELT translator.

When GCC will provide additional hooks for plugins, making them available to MELT code should hopefully be quite easy.

\section{Pattern matching in MELT}

Pattern matching [12, 14, 18, 30] is an essential operation in symbolic processing and formal handling of programs, and is one of the buying features of high-level programming languages (notably Ocaml and Haskell). Several tasks inside GCC are mostly pattern matching (like simplification and folding of constant expressions ${ }^{41}$. Code using MELT pattern matching facilities is much more concise than its (generated or even hand-written) $C$ equivalent.

\subsection{Using patterns in MELT}

Developers using MELT often need to filter complex GCC stuff (in particular gimple or tree-s) in their GCC passes coded in MELT. This is best achieved with pattern matching. The matching may fail (if the data failed to pass the filter) or may extract information from the matched data.

\footnotetext{
${ }^{39}$ Each bound name is bound only once, and there are no separate namespaces like in C or Common Lisp.

${ }^{40}$ Like (current_module_environment_container) and (parent_module_environment), etc.

${ }^{41}$ Strangely, GCC has several specialized code generators, but none for pattern matching: so the file gcc/fold-const.c is hand-written (16KLOC).
} 


\subsubsection{About pattern matching}

Patterns are major syntactic constructs (like expressions and let-bindings in Scheme or MELT). In MELT, a pattern starts with a question mark, which is parsed particularly: ? $\mathrm{x}$ is the same as (question $\mathrm{x}$ ) [it is the pattern variable $x] . ?_{-}$is ${ }^{42}$ the wildcard pattern (matching anything). An expression occurring in pattern context is a constant pattern. Patterns may be nested (in composite patterns) and occur in match expressions.

Elementary patterns are ultimately translated into code that tests that the matched thing $\mu$ can be filtered by the pattern $\pi$ followed by code which extracts appropriate data from $\mu$ and fills some locals with information extracted from $\mu$. Composite patterns need to be translated and optimized to avoid, when possible, repetitive tests or fills.

\subsubsection{An example of pattern usage in gcc $^{\text {melt }}$}

Many tasks depend upon the form of [some intermediate internal representation of] user source code, and require extracting some of its sub-components. For instance, the author has written (in a single day) a GCC extension in MELT to check simple coding rules in melt-runtime.c, (e.g., in function of figure 2). When enabled with -fplugin-melt-arg-mode=meltframe, it adds a new pass (after the "ssa" pas 43 of GCC [21]) melt_frame_pass to GCC. This pass first finds the declaration of the local meltfram_- in the following pass execute function:

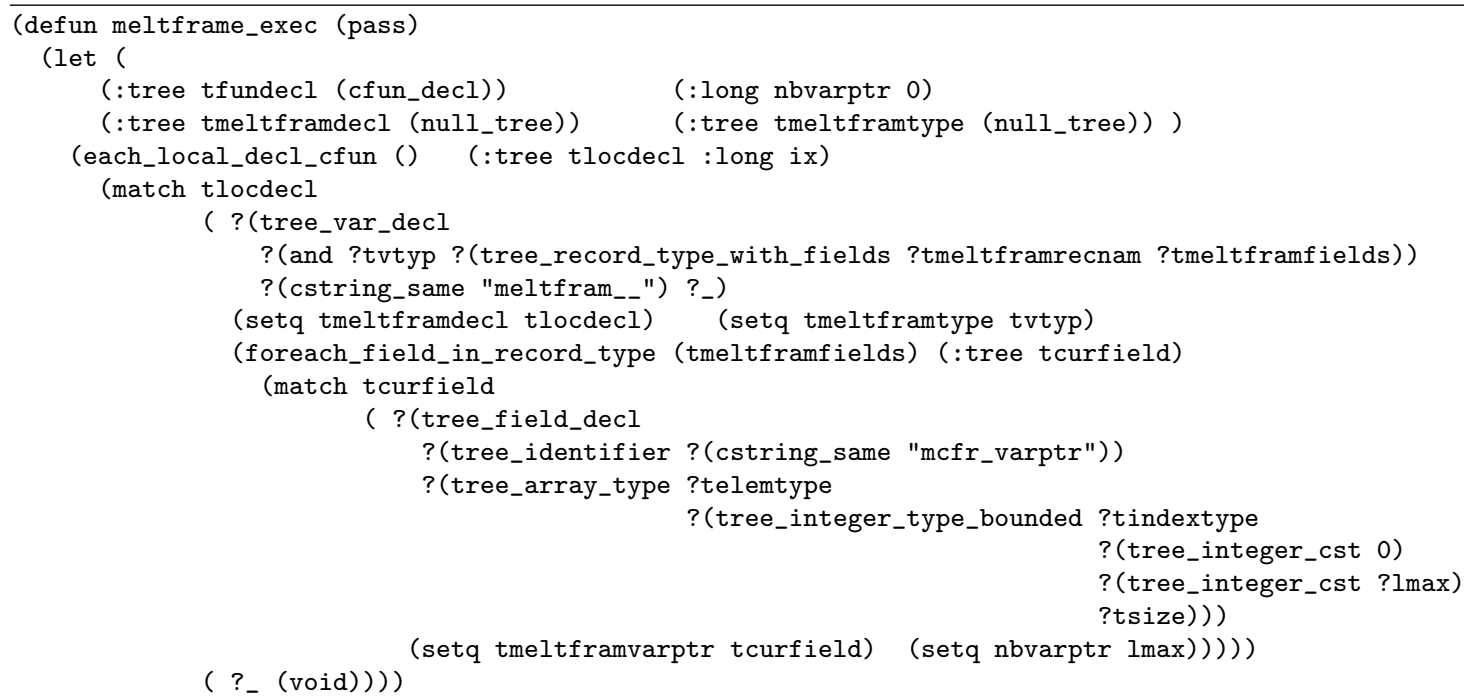

The let line 2 spans the entire MELT function meltframe_exec, with bindings lines $3 \& 4$ for tfundecl, nbvarptr, tmeltframdecl \& tmeltframtype locals. The each_local_decl_cfun is a citerator (iterating -lines 5 to 11- on the Tree-s representing the local declarations in the function). The match expression filters the current local declaration tlocdecl (lines 7-11). When it is a variable declaration (line 7) whose type matches the sub-pattern line 8 and whose name (line 9) is exactly meltfram_-, we assign (line 10) appropriately tmeltframdecl \& tmeltframtype, and we iterate (line 11) on its fields to find, by the match (lines 12-21), the declaration of field mcfr_varptr (in the

\footnotetext{
${ }^{42} ?_{-}$can be pronounced as "joker"

${ }^{43}$ ssa means Static Single Assignment, so at that stage the code is represented in Gimple/SSA form, so each SSA variable is assigned once!
} 
$C$ code), and its array index upper bound lmax, assigning them (line 20) to locals tmeltframvarptr \& nbvarptr. Otherwise, using the wildcard pattern ?,, we give a :void result for the match of tlocdecl (line 21).

Once the declaration of meltfram ${ }_{--}$and of its mcfr_varptr field has been found ${ }^{44}$ in the current function (given by cfun inside GCC), we iterate on each basic block bb of that function, and on each gimple statement $g$ of that basic block, and we match that statement $g$ to find assignments to or from meltfram ${ }_{--}$mcfr_varptr $[\kappa]$ where $\kappa$ is some constant integer index:

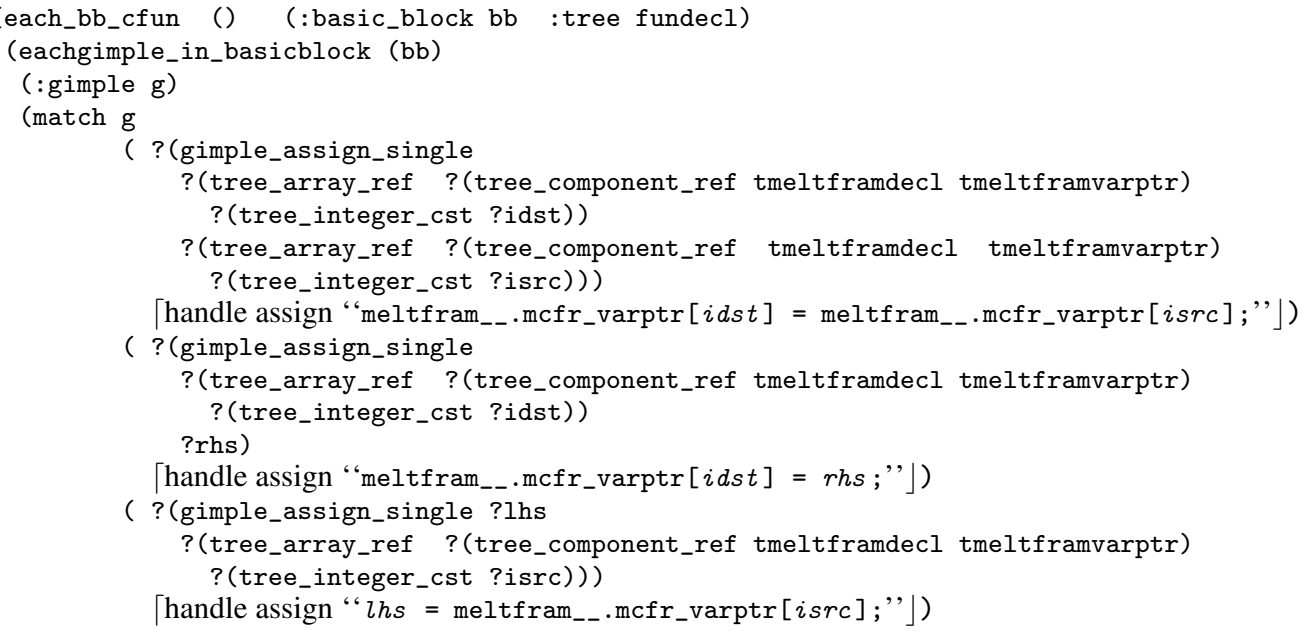

The gimple $\mathrm{g}$ is matched against the most filtering pattern (lines 26-30, for assignments like "meltfram_. $\left.\mathrm{mcfr}_{-} \operatorname{varptr}[i d s t]=\operatorname{meltfram} \mathrm{m}_{-} \cdot \operatorname{mcfr}_{-} \operatorname{varptr}[i s r c] ; "\right)$ first, then against the more general patterns -for "meltfram_-. mcfr_varptr $[i d s t]=r h s$;" where $r h s$ is any simple operandlines 32-36, and for " $l h s=\operatorname{meltfram}$-. $_{\text {. }} \operatorname{mcf} r_{-} \operatorname{varptr}[i s r c]$;" lines 37-40. The MELT programmer should order his matching clauses from the more specific to the more general.

Other code (not shown here) in function meltframe_exec remembers all left-hand side and right-hand side occurences of meltfram_.. mcfr_varptr $[\kappa]$, and issues a warning when such a slot is not used.

We see that a match is made of several match-cases, tested in sequence until a match is found. Each case starts with a pattern, followed by sub-expressions which are computed with the pattern variables of the case set appropriately by the matching of the pattern; the last such sub-expression is the result of the entire match. Like other conditional forms in MELT, match expressions can give any thing (stuff, e.g., : long ... or even :void, or value) as their result. Patterns may be nested like the tree_var_decl or tree_record_type above. All the locals for pattern variables in a given match-case are cleared (before testing the pattern). It is good style to end a match with a catch-all wildcard ? - pattern.

A pattern is usually composite (with nested sub-patterns) and has a double role: first, it should test if the matched thing fits; second, when it does, it should extract things and transmit them to eventual sub-patterns; this is the fill of the pattern. The matching of a pattern should conventionally be without side-effects (other than the fill, i.e. the assignment of pattern variables).

Patterns may be non-linear: in a matching case, the same pattern variable can occur more than once; then it is set at its first occurrence, and tested for identity ${ }^{45}$ with $==$ in the generated $C$ code on all

\footnotetext{
${ }^{44} \mathrm{~A}$ warning is issued if meltfram_- or mcfr_varptr has not been found.

${ }^{45}$ We don't test for equality of values or other things, knowing that $\lambda$-term equality is undecidable, and acknowledging that deep equality compare of ASTs like tree or gimple is too expensive.
} 
the following occurrences. This is useful in patterns like ? (gimple_assign_single ?var ?var) to find assignments of a variable var to itself.

\subsection{Pattern syntax overview}

A pattern $\pi$ may match some matched thing $\mu$, or may fail. It the matching succeeds, sub-patterns may be matched, and pattern variables may become bound. The thing bound by some pattern variable is checked in following occurrences of the same pattern variables and is available inside the match-clause body.

Patterns may be one of:

- expressions $\varepsilon$ (e.g., constant literals) are (degenerated) patterns. They match the matched data $\mu$ iff $\varepsilon$ $==\mu$ (for the $\mathrm{C}$ sense of equality, which for pointers is their identity).

- The wildcard noted ?_ matches everything (every value or stuff) and never fails.

- a pattern variable ? $v$ matches $\mu$ if it was unset (by a previous [sub-]matching of the same ? $v$ ). In addition, it is then bound to $\mu$. If the pattern variable was previously set, it is tested for identity (with equality in the $\mathrm{C}$ sense).

- most patterns are matcher patterns ? $\left(\begin{array}{lllllll}m & \varepsilon_{1} & \ldots & \varepsilon_{n} & \pi_{1} & \ldots & \pi_{p}\end{array}\right)$ where the $n \geq 0$ expressions $\varepsilon_{i}$ are input parameters to the matcher $m$ and the $\pi_{j}$ sub-patterns are passed extracted data. The matcher is either a $c$-matcher (declaring how to translate that pattern to $C$ code) or it is a funmatcher (matching is done by a MELT function returning secondary things).

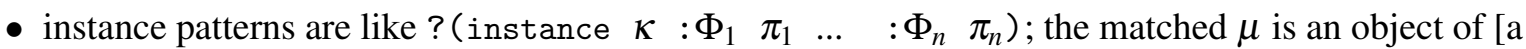
sub-] class $\kappa$ whose field $\Phi_{i}$ matches sub-pattern $\pi_{i}$.

- conjunctive patterns are ? (and $\pi_{1} \quad \ldots \quad \pi_{n}$ ) and they match $\mu$ iff every $\pi_{i}$ in sequence matches $\mu$; notice that when some $\pi_{i}$ is a pattern variable ? $v$ that variable is matched and $\mu$ should match the further $\pi_{j}$ (with $j>i$ ) with $v$ appropriately bound to $\mu$. (This generalizes the as keyword inside Ocaml patterns).

- disjunctive patterns are ? (or $\pi_{1} \quad \ldots \quad \pi_{n}$ ) and they match $\mu$ if one of the $\pi_{i}$ matches $\mu$.

\subsection{C-matchers and fun-matchers}

The c-matchers are one of the building blocks of patterns - much like primitives are one of the building blocks of expressions. Like primitives, c-matchers are defined as a specialized $\mathrm{C}$ code generation template. In the example above (\$4.1.2), most composite patterns involve c-matchers: tree_var_decl, tree_record_type and cstring_same are C-matchers.

Like for every pattern, a C-matcher defines how the pattern using it should perform its test, and then how it should do its fill. A simple example of a C-matcher is cstring_same: some :cstring stuff $\sigma$ matches the pattern? (cstring_same "fprintf") iff $\sigma$ is the same as the const char* string "fprintf" given as input to our c-matcher. This c-matcher has a test part, but no fill part (because used without sub-patterns).

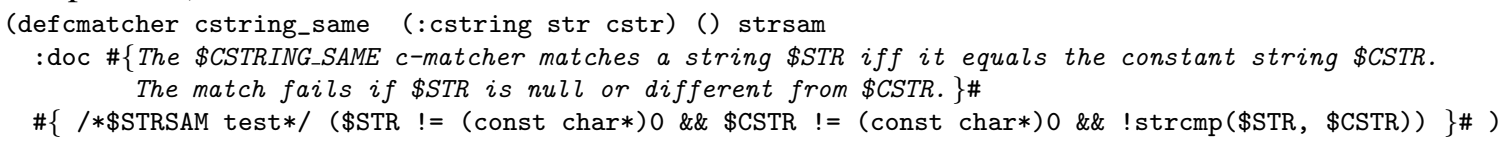

Notice that the state symbol strsam is used inside a comment, to uniquely identify each occurrence in the generated $\mathrm{C}$, and that we take care of testing against null const char* pointers to avoid crashes.

A more complex (and GCC specific) example is the gimple_assign_single c-matcher (to filter single assignments in compiled code). It defines both a testing and a filling expansion using two macro-strings: 


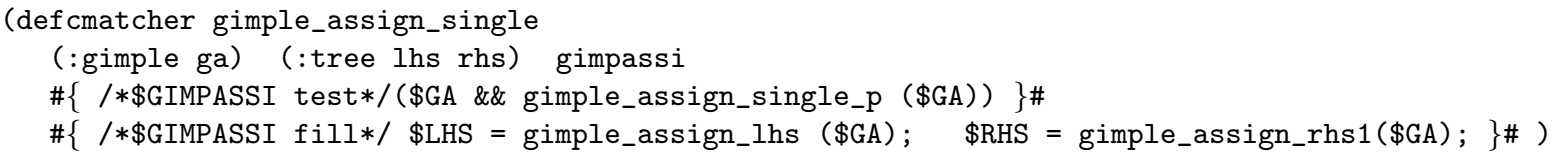

Here ga is the matched gimple, and lhs \& rhs are the output formals: they are assigned in the fill expansion to transmit tree-s to sub-patterns!

C-matchers are a bit like Wadler's notion of Views [30], but are expanded into C code. MELT also has fun-matchers which similarly are views defined by a MELT function returning a non-nil value if the test succeeded with several secondary results giving the extracted things to sub-patterns. For example the following code defines a fun-matcher isbiggereven ${ }^{46}$ such that the pattern ? (isbiggereven $\mu \pi$ ) matches a : long stuff $\sigma$ iff $\sigma$ is a even number, greater than the number $\mu$, and $\sigma / 2$ matches the subpattern $\pi$. We define an auxiliary function matchbiggereven to do the matching [we could have used a lambda]. If the match succeeds, it returns a true (i.e. non nil) value (here fmat) and the integer to be matched with $\pi$. Its first actual argument is the fun-matcher isbiggereven itself. The testing behavior of the matching function is its first result (nil or not), and the fill behavior is through the secondary results.

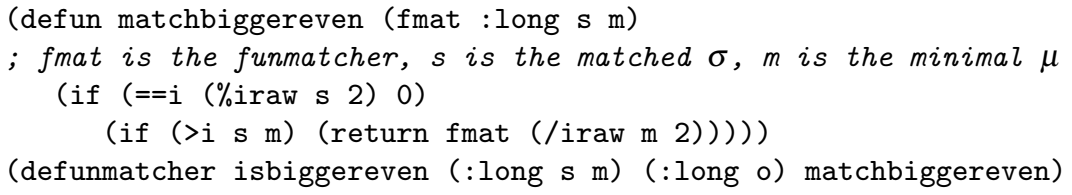

The fun-matcher definition has an input formals list and an output formal list, together defining the expected usage of the fun-matcher operator in patterns.

Both c-matchers and fun-matchers can also define what they mean in expression context (not in pattern one). So the same name can be used for constructing expressions and for destructuring patterns.

\subsection{Implementing patterns in MELT}

Designing and implementing patterns in MELT was quite difficult, because a good translation of pattern matching should :

- factorize, when possible, common sub-patterns, to avoid testing twice the same thing.

- share, when appropriate, data extracted from subpatterns.

- preferably re-use the many temporary locals used by the translation of the match, to lower the current MELT stack frame size.

Our first implementation of pattern translation to $\mathrm{C}$ is quite naive, and uses simple memoization techniques to factorize sub-patterns or share extracted data.

A better implementation of the pattern translator builds explicitly a directed graph (with shared nodes for tests and data), like figure 4. The graph has data nodes (for temporary variables for [sub-]matched things, or for boolean flags internal to the match) and elementary control steps. These steps are either tests (with both a "then" and an "else" jumps to other steps) or computations (usually with a single jump to a successor step). Some steps just set an internal boolean flag, or compute the conjunction of other flags. Other steps represent the testing or the filling parts of c-matchers or fun-matchers. Final success steps correspond to sub-expressions in the body of the matched clause and are executed if a flag is set.

\footnotetext{
${ }^{46}$ Our isbiggereven could also be defined as a c-matcher!
} 
For instance a simple match (where $v$ is the matched value) like below is translated into the complex internal graph ${ }^{47}$ given in figure 4.

(match $\mathrm{v}$

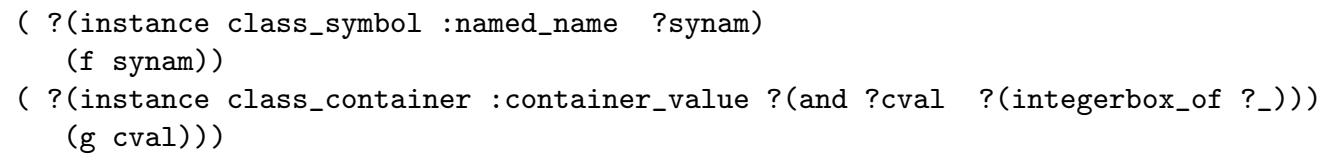

A more complex match like (match tcurfield ...) of 4.1 .2 code line 12-20 produces about 20 match steps and 12 match data. This enhanced pattern matching is not entirely implemented at time of writing: the generation of the control graph for the match is implemented, but its translation into $\mathrm{C}$ is incomplete.

\section{Conclusions and future work}

Enhancing a legacy huge software with a domain specific language or scripting language is always a major challenge (\$1), since incorporating a DSL inside a software is a major architectural design decision which should be taken early. Mature big software like GCC have their coding habits, memory management strategies and data organization which makes it very difficult to embed an existing scripting language (like Python, Ocaml, Ruby, ...).

We have shown that adding a high-level DSL to a big software like GCC is still possible, by designing a run-time system $\$ 2$ compatible with the existing infrastructure (notably $\mathrm{Gg}-\mathrm{c}$ ) and most importantly, by having the DSL deal both with boxed values and raw existing stuff in 3.2 . Translating the DSL to the language (with its habits) used in that big software ( $C$ for GCC) enables high-level language constructs in our DSL. We have described a set of language constructs in $\$ 3.4$ (c-matchers, primitives, c-iterators, ...) which give templates for $C$ code generation.

Our empirical approach of designing and implementing a DSL like MELT to fit into a large software like GCC, could probably be re-used for adding DSLs inside other huge mature software projects: designing a runtime suitable for such a project, having several sorts of things (values and stuff), generating code in the style of the existing legacy, and defining adequate language constructs giving code-generating templates.

Future work within MELT is mostly using this DSL to build interesting GCC extensions. P. Vittet has started in May 2011 a Google Summer of Code project to add specific warnings into GCC using MELT. A. Lissy considers using it for Linux kernel [13] code analysis. The opengpu mode should be completed. Also, some language features can be added or improved:

1. variadic functions, possibly provided by a :rest keyword similar to Common Lisp's \&rest. These should be very useful for debugging and tracing messages.

2. adding backtracking or iterating pattern constructs; for instance to be able to have a pattern for any :gimple_seq stuff containing at least one gimple matching a given sub-pattern.

3. adding a nice usable and hygenic macro system, inspired by Scheme's def syntax

4. performance improvements might be achieved by sometimes translating MELT function calls into a $\mathrm{C}$ function call whose signature mimicks the MELT function signature.

\footnotetext{
${ }^{47}$ To debug the pattern-match translator, MELT is generating a graph to be displayed with GraphViz. We have edited it (by removing details like source code location) for clarity.
} 


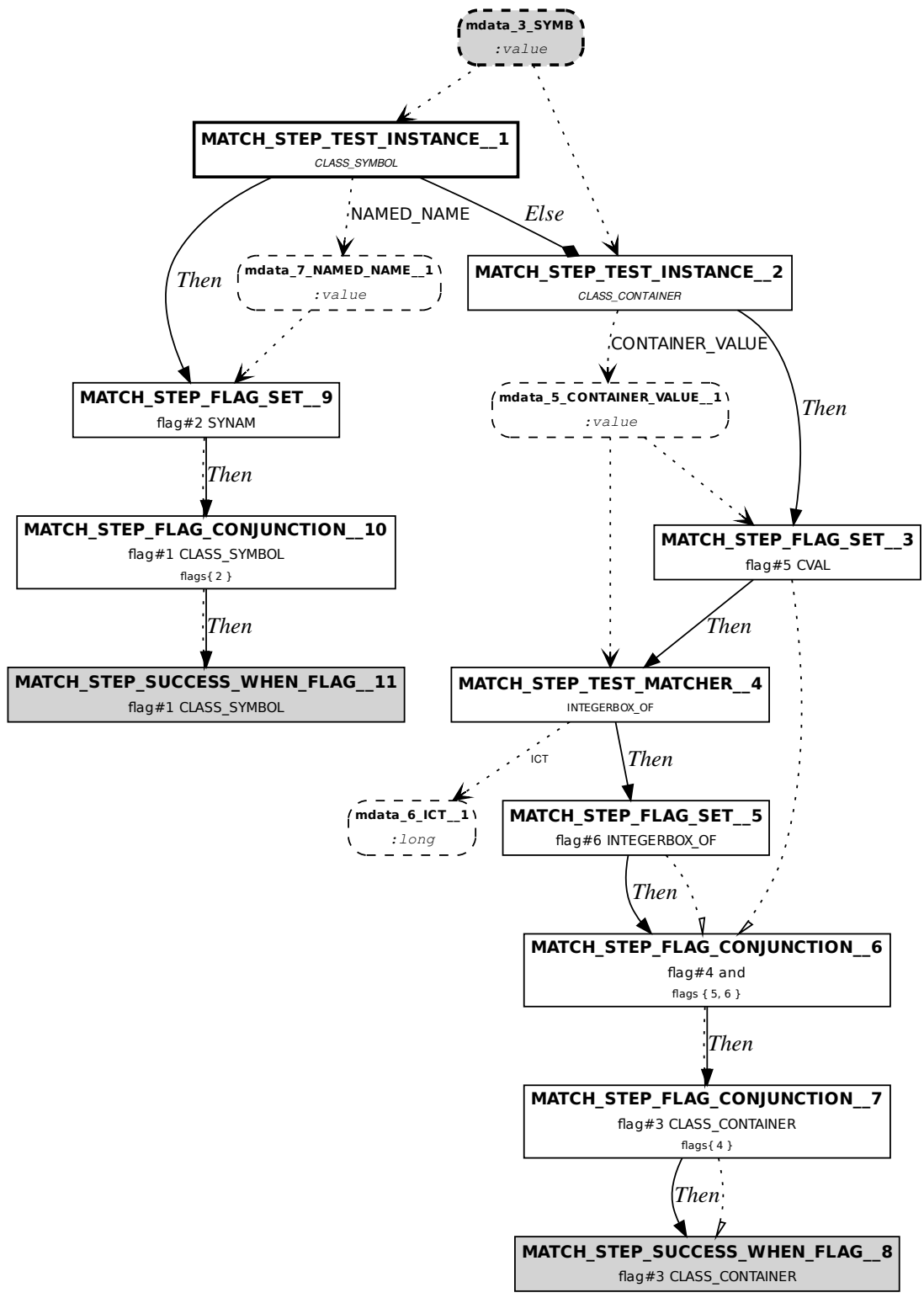

Legend:

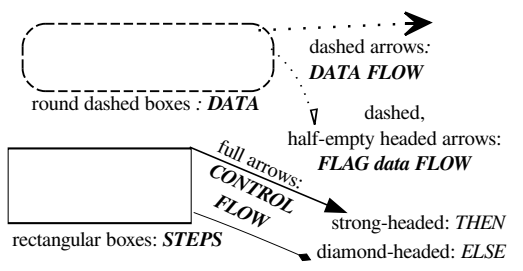

Figure 4: internal graph for match 
5. a message caching machinery, where every MELT message passing occurrence would use a cache (keeping the last class of the sending).

6. a central monitor, which would communicate with parallel $\mathrm{GCC}^{\text {melt }}$ compilations through asynchronous textual protocols.

More generally, making MELT more high-level and more declarative (in J.Pitrat's [19, 20] sense) to be able to express GCC passes easily and concisely is an interesting challenge, and could be transposed to other legacy software.

\section{Acknowledgments}

Work on MELT has been funded by DGCIS thru ITEA GlobalGCC and FUI OpenGPU projects.

Thanks to Albert Cohen, Jan Midtgaard, Nic Volanschi and to the anonymous reviewers for their constructive suggestions and their proof-reading. Residual mistakes are mine.

\section{References}

[1] P. Collingbourne \& P. Kelly (2009): A Compile-Time Infrastructure for GCC Using Haskell. In: GROW09 workshop, within HIPEAC09, http://www.doc.ic.ac.uk/ phjk/GROw09/, Paphos, Cyprus.

[2] P. Cousot \& R. Cousot (1992): Abstract Interpretation Frameworks. J. Logic and Computation 2(4), pp. 511-547, doi:10.1093/logcom/2.4.511.

[3] P. Cousot \& R. Cousot (2004): Basic Concepts of Abstract Interpretation, pp. 359-366. Kluwer Academic Publishers, doi:10.1007/978-1-4020-8157-6_27

[4] P. Cousot, R. Cousot, J. Feret, L. Mauborgne, A. Miné, D. Monniaux \& X. Rival (2005): The ASTRÉE Analyser. In: Proc. ESOP'05, LNCS 3444, Edinburgh, Scotland, pp. 21-30, doi:10.1007/978-3-540-31987$0 \_3$.

[5] P. Cousot, R. Cousot, J. Feret, L. Mauborgne, A. Miné, D. Monniaux \& X. Rival (2006): Combination of Abstractions in the ASTRÉE Static Analyzer. In M. Okada \& I. Satoh, editors: Eleventh Annual Asian Computing Science Conference (ASIAN'06), 4435, Springer, Berlin, Tokyo, Japan, LNCS, pp. 124, doi: $10.1007 / 978-3-540-77505-8 \_23$.

[6] D. Engler, B. Chelf, A. Chou \& S. Hallem (2000): Checking system rules using system-specific, programmerwritten compiler extensions. In: Proceedings of the 4th conference on Symposium on Operating System Design \& Implementation - Volume 4, OSDI'00, USENIX Association, Berkeley, CA, USA, pp. 1-16.

[7] C. Flanagan, A. Sabry, B. F. Duba \& M. Felleisen (2004): The essence of compiling with continuations. SIGPLAN Not. 39, pp. 502-514, doi $10.1145 / 989393.989443$.

[8] GCC community (2011): GCC internals doc. Available at http://gcc.gnu.org/onlinedocs/gccint/.

[9] T. Glek \& D. Mandelin (2008): Using GCC instead of Grep and Sed. In: GCC Summit 2008, Ottawa, pp. 21-32.

[10] D. Guilbaud, E. Goubault, A. Pacalet, B. Starynkévitch \& F. Védrine (2001): A Simple Abstract Interpreter for Threat Detection and Test Case Generation. In: WAPATV'01, with ICSE'01, Toronto.

[11] R. Jones \& R. D. Lins (1996): Garbage Collection: Algorithms for Automatic Dynamic Memory Management. Wiley.

[12] F. Le Fessant \& L. Maranget (2001): Optimizing Pattern-Matching. In: Proc. 2001 ICFP, ACM Press.

[13] A. Lissy (2011): Model Checking the Linux Kernel (FOSDEM2011).

[14] L. Maranget (2007): Warnings for pattern matching. J. Functional Programming 17. 
[15] L. Maranget (2008): Compiling Pattern Matching to Good Decision Trees .

[16] G. Marpons-Ucero, J. Mariño-Carballo, M. Carro, Á. Herranz-Nieva, J. J. Moreno-Navarro \& L. Fredlund (2008): Automatic Coding Rule Conformance Checking Using Logic Programming. In: PADL, pp. 18-34, doi:10.1007/978-3-540-77442-6_3.

[17] B. Monate \& J. Signoles (2008): Slicing for Security of Code. In: TRUST, pp. 133-142, doi:10.1007/978-3540-68979-9_10

[18] J. Pitrat (1995): Speaking about and Acting upon Oneself. Technical Report 1995/29, LIP6/Laforia.

[19] J. Pitrat (1996): Implementation of a reflective system. Future Gener. Comput. Syst. 12(2-3), pp. 235-242, doi $10.1016 / 0167-739 X(96) 00011-8$

[20] J. Pitrat (2009): Artificial Beings (the conscience of a conscious machine). Wiley / ISTE, doi $10.1002 / 9780470611791$

[21] S. Pop (2006): The SSA Representation Framework: Semantics, Analyses and GCC Implementation. Ph.D. thesis, Ecole des Mines de Paris. Available at http://www.cri.ensmp.fr/classement/doc/A-381.pdf.

[22] C. Queinnec (1996): Lisp in Small Pieces. Cambridge Univ. Pr., New York, NY, USA.

[23] N. Ramsey \& S.P. Jones (2000): A single intermediate language that supports multiple implementations of exceptions. In: Proc. PLDI '00, ACM, New York, NY, USA, pp. 285-298, doi:10.1145/349299.349337.

[24] B. Starynkevitch (2006-2011): MELT code [GPLv3] within GCC. http://gcc-melt.org/ and svn://gcc.gnu. org/svn/gcc/branches/melt-branch

[25] B. Starynkevitch (2007): Multi-Stage Construction of a Global Static Analyzer. In: GCC Summit 2007, Ottawa, pp. 143-156.

[26] B. Starynkevitch (2009): Middle End Lisp Translator for GCC, achievements and issues. In: GROW09 workshop, within HIPEAC09, http://www.doc.ic.ac.uk/ phjk/GROw09/, Paphos, Cyprus.

[27] K. Trifunovic, A. Cohen, D. Edelsohn, F. Li, T. Grosser, H. Jagasia, R. Ladelsky, S. Pop, J. Sjödin \& R. Upadrasta (2010): GRAPHITE Two Years After: First Lessons Learned From Real-World Polyhedral Compilation. In: GCC Research Opportunities Workshop (GROW'10), Pisa Italie. Available at http://hal.inria.fr/inria-00551516/en/.

[28] A. Venet \& G. Brat (2004): Precise and efficient static array bound checking for large embedded C programs. In: PLDI '04: Proceedings of the ACM SIGPLAN 2004 conference on Programming language design and implementation, ACM Press, New York, NY, USA, pp. 231-242, doi $10.1145 / 996841.996869$.

[29] N. Volanschi (2006): A Portable Compiler-Integrated Approach to Permanent Checking. In: ASE '06: Proceedings of the 21st IEEE International Conference on Automated Software Engineering (ASE'06), IEEE Computer Society, Washington, DC, USA, pp. 103-112, doi:10.1109/ASE.2006.8.

[30] P. Wadler (1987): Views: a way for pattern matching to cohabit with data abstraction. In: Proc. POPL '87, ACM, New York, NY, USA, pp. 307-313, doi:10.1145/41625.41653 